1 RH: Phylogenetic biome shifts among paleobiomes

2

\title{
Modeling phylogenetic biome shifts on a planet with a past
}

${ }^{1}$ Department of Biology, Washington University in St. Louis, One Brookings Drive, 
13 Abstract. - The spatial distribution of biomes has changed considerably over deep time, so

14 the geographical opportunity for an evolutionary lineage to shift into a new biome may

15 depend on how the availability and connectivity of biomes has varied temporally. To better

16 understand how lineages shift between biomes in space and time, we developed a

17 phylogenetic biome shift model in which each lineage shifts between biomes and disperses

18 between regions at rates that depend on the lineage's biome affinity and location relative to

19 the spatiotemporal distribution of biomes at any given time. To study the behavior of the

20 biome shift model in an empirical setting, we developed a literature-based representation

21 of paleobiome structure for three mesic forest biomes, six regions, and eight time strata,

22 ranging from the Late Cretaceous (100 Ma) through the present. We then fitted the model

23 to a time-calibrated phylogeny of 119 Viburnum species to compare how the results

24 responded to various realistic or unrealistic assumptions about paleobiome structure.

25 Ancestral biome estimates that account for paleobiome dynamics reconstructed a warm

26 temperate (or tropical) origin of Viburnum, which is consistent with previous fossil-based

27 estimates of ancestral biomes. Imposing unrealistic paleobiome distributions led to

28 ancestral biome estimates that eliminated support for tropical origins, and instead inflated

29 support for cold temperate ancestry throughout the warmer Paleocene and Eocene. The

30 biome shift model we describe is applicable to the study of evolutionary systems beyond

31 Viburnum, and the core mechanisms of our model are extensible to the design of richer

32 phylogenetic models of historical biogeography and/or lineage diversification. We

33 conclude that biome shift models that account for dynamic geographical opportunities are

34 important for inferring ancestral biomes that are compatible with our understanding of

35 Earth history. 
bioRxiv preprint doi: https://doi.org/10.1101/832527; this version posted June 1, 2020. The copyright holder for this preprint (which was not certified by peer review) is the author/funder, who has granted bioRxiv a license to display the preprint in perpetuity. It is made available under aCC-BY 4.0 International license.

36 (Keywords: phylogenetics, ancestral states, biome shifts, niche conservatism, historical

37 biogeography) 
Biomes are ecologically and climatically distinct species assemblages that vary in

40 size, shape, and continuity across geographical regions, in large part due to regional

41 differences in temperature, precipitation, seasonality, altitude, soil types, and

42 continentality (Whittaker 1970; Wolfe 1985; Olson et al. 2001; Mucina 2019). The diversity

43 of biomes occupied by particular lineages also varies considerably, with some clades

44 exhibiting strict associations with particular biomes, and others showing multiple

45 transitions between biomes over time (Donoghue and Edwards 2014). Although it is

46 accepted that clade-wide variation in regional biome occupancy was generated and is

47 maintained by evolutionary forces including speciation, extinction, dispersal, and

48 adaptation to new biomes, it remains difficult to estimate exactly when, where, and under

49 what conditions phylogenetic lineages first shifted into the biomes that their descendants

50 inhabit today.

51 In current practice, ancestral regions and biome affinities are often estimated

52 independently of one another, and then relationships between regions and biomes are

53 compared post hoc (e.g., Crisp et al. 2009; Weeks et al. 2014). Although such studies yield

54 important evolutionary insights, the estimates themselves do not account for how lineages

55 might move between regions or adapt to newly encountered biomes given the temporally

56 variable spatial configuration of biomes across regions. Conceptually, how a biome is

57 geographically distributed should influence how easily a lineage might disperse into a new

58 region or shift into a new biome, an effect Donoghue and Edwards (2014) termed

59 geographical opportunity. One strategy to model the effect of geographical opportunity

60 first defines discrete regions that are exactly coincident with modern day biomes, and then 
61 assumes that species within a given region occur within the corresponding biome. Cardillo

62 et al. (2017) carried out such an analysis in studying the biogeography of the Australian

63 plant clade, Hakea (Protaceae), using method features developed by Matzke (2014), where

64 total regional area and shared perimeter lengths tuned dispersal rates between bioregions.

65 This innovative strategy depends crucially on the uniformity of biome composition within

66 each region. Larger, discrete regions may very well be dominated by a single biome type,

67 yet still be composed of assorted dominant, subdominant, and marginal biome types at

68 local scales.

69 More importantly for our purposes, defining geographical opportunity based on

70 modern biome features (such as area and shared perimeter), may be problematic in

71 instances where the spatial distribution of biomes has changed considerably over time,

72 since those changes should also influence when and where ancestral lineages shift between

73 regions and/or biomes. For example, if woodlands dominated a particular region until the

74 rise of grasslands, that might inform when a grassland-adapted lineage first dispersed into

75 that region. That is, if the presences or absences of biomes among regions influence

76 modern species ranges, then temporal variation in regional biome availability should

77 similarly influence our models of range evolution.

78 To model how paleoecological dynamics might influence range evolution, Meseguer

79 et al. (2015) fitted ecological niche models (ENMs) to fossil data so as to limit the

80 connectivity between regions for biogeographical models that estimate ancestral ranges

81 (Ree and Smith 2008). While this strategy is quite promising, its current form requires that

82 the clade under study (Hypericum of Hypericaceae, in their case) has a sufficiently rich

83 fossil record over space and time to inform the ENM. It also assumes that all lineages face 
84 the same, broad ecological limitations to range evolution, independent of what particular

85 biome affinity each lineage possesses at a given moment. Although the quality of the fossil

86 record is largely out of our control, the second assumption could be relaxed: ideally, if a

87 clade contains sub-lineages that specialize in both woodland and in grassland habitats, any

88 particular lineage's range should be principally limited by the availability of the specific

89 biome to which that lineage is adapted, rather than being constrained based on a broader,

90 clade-wide average of grassland and woodland lineages.

91 In this paper, we aim to address the aforementioned challenges facing current

92 phylogenetic models of biome shifting by incorporating four key properties: (1) that biome

93 shifts and dispersal events share a common state space over biomes and regions, (2) that

94 any discrete region may contain a number of different biomes, (3) that the geographical

95 structure of biomes within and between regions can vary over time, and (4) that lineages

96 adapted to different biomes and located in different regions will experience different

97 dispersal rates between regions and different shift rates into new biomes. We begin by

98 introducing a graph-based approach to characterize the availability, prevalence, and

99 connectivity of regional biomes through time, building on the framework introduced by

100 Landis (2017). We then develop an event-based evolutionary process using a time-

101 stratified continuous-time Markov chain that models biome shifts and dispersal given the

102 ways in which biome distributions have changed over time. Because the exact influence of

103 extrinsic geographical factors and/or ecological structure is bound to vary from clade to

104 clade, the degree of influence of such features on the evolutionary model are treated as free

105 parameters to be estimated from the data itself. 
To explore the possible importance of paleobiome structure on lineage movements

107 among biomes, we apply our model to Viburnum, a clade of $\sim 165$ species that originated in

108 the Late Cretaceous and are today found in tropical, warm temperate, and cold temperate

109 forests throughout Eurasia and the New World. We generated paleobiome graphs for these

110 three mesic forest biomes across six continental regions for eight major time intervals over

111 the past hundred million years. Fitting the model to our Viburnum dataset all-but-

112 eliminates the possibility of a cold temperate origin of the clade. This is consistent with our

113 understanding of the important biogeographic role of a "boreotropical" flora (see below)

114 during the Paleocene and Eocene, and with our recent fossil-based ancestral biome

115 estimates in Viburnum (Landis et al. 2020).

METHODS

118 Viburnum (Adoxaceae) is a clade of $\sim 165$ extant plant species that originated just

119 before the Cretaceous-Paleogene (K-Pg) boundary, roughly $70 \mathrm{Ma}$. Previous studies of

120 phylogenetic relationships (Clement et al. 2014; Spriggs et al. 2015; Eaton et al. 2017) and

121 divergence times (Spriggs et al. 2015; Landis et al. 2020) provide a firm basis for

122 understanding the order and timing of lineage diversification events in Viburnum. In this

123 study, we focus on a subsample of 119 Viburnum species with relationships that are highly

124 supported by phylogenomic data (Eaton et al. 2017; Landis et al. 2020) and whose

125 divergence times were time-calibrated under the fossilized-birth death process (Heath et

126 al. 2014) as described in Landis et al. (2020).

127 Viburnum species occupy six continental-scale regions: Southeast Asia, including the

128 Indoaustralian Archipelago and the Indian subcontinent; East Asia, including China, 
129 Taiwan, and Japan; Europe, including the North African coast, portions of the Middle East,

130 and the Azores and the Canary Islands; a North American region north of Mexico; a Central

131 American region that includes Mexico, Cuba, and Jamaica; and the South American Andes.

132 The choice of these biogeographic regions is discussed in more detail in Landis et al.

133 (2020). Briefly, these regions were selected because: (1) each is an area of Viburnum

134 species/clade endemism; (2) there are no individual Viburnum species that occur in more

135 than one of these regions; and (3) there are significant barriers to the migration of

136 viburnums between these regions today and in the relevant past. As a concrete example,

137 consider our recognition of North America and of Mexico, Central America, and the

138 Caribbean as two distinct biogeographic regions for Viburnum. Multiple Viburnum species

139 are endemic to the forests in each of these regions, and none of these species spans

140 between them. Importantly, for mesic forest plants such as Viburnum, these two regions

141 are entirely separated from one another by an extensive zone of low-lying arid lands in

142 southeastern Texas and northeastern Mexico, with vegetation generally classified as xeric

143 shrubland or mesquite-chaparral savanna. These areas are uninhabitable by viburnums

144 today, and probably have not been accessible to these plants since widespread drying and

145 the spread of C4 grasses commenced by at least the late Miocene (e.g., Godfrey et al. 2018).

146 Using our criteria, the most difficult division is between the southeast Asian and eastern

147 Asian regions. Although these do both harbor endemic species/clades, and these species

148 are adapted to different environmental conditions, there are now, and have been in the

149 past, opportunities for north-south migration between mesic forests of the two regions.

150 Finally, we note that our biogeographic regions are not strictly defined in terms of tectonic

151 plates as they do not determine the movements and distributions of Viburnum. Thus, based 
152 on the criteria above, we split the vast Eurasian plate into a European region and two Asian

153 regions. Likewise, the boundary between the North American and the Caribbean plates

154 appears to have had no impact on Viburnum movements as there are individual species

155 (e.g., V. hartwegii) that span the two.

156 Across those regions, living viburnums are affiliated with mesic forest biomes and

157 show widespread parallel evolution of leaf form, leafing habit, and physiology coincident

158 with transitions between warmer and colder biomes (Schmerler et al. 2012; Chatelet et al.

159 2013; Spriggs et al. 2015; Scoffoni et al. 2016; Edwards et al. 2017). Five extinct Viburnum

160 lineages are known by their fossil pollen grains recovered from North American and

161 European locales. Four of these fossils are older samples (48 to $33 \mathrm{Ma}$ ) from paleofloral

162 communities that we previously judged to be warm temperate or subtropical (Landis et al.

163 2020). For our analyses in this study, we defined three mesic forest biomes based on

164 annual temperatures and rainfall patterns (Edwards et al. 2017). Tropical forests have high

165 temperatures and precipitation year-round, showing little seasonality. Warm temperate

166 forests, which include paratropical, lucidophyllous, and cloud forests, vary seasonally in

167 temperature and precipitation, but do not experience prolonged freezing temperatures

168 during the coldest months. Cold temperate forests also experience seasonal temperatures

169 and precipitation, but average minimum temperatures drop below freezing in at least one

170 of the coldest months.

171 Because we are interested in how biome states and regional states evolve in tandem,

172 we constructed a set of $3 \times 6=18$ compound states that we call biome-region states.

173 Throughout the paper, we identify the biome-region state for a lineage in biome state $i$ and

174 region state $k$ with the notation $(i, k)$. However, in practice, we encode biome-region states 
175 as integers with values from 1 to 18. Biome-region state codings for Viburnum are

176 translated from Landis et al. (2020), though here we combine cloud forests and warm

177 temperate forests into a single warm temperate category.

178 All of the 119 Viburnum species we included in our analysis are found in a single

179 biome, except for two East Asian (V._chinshanense and V._congestum) and one North

180 American (V. rufidulum) species, which reside in forests possessing both warm and cold

181 temperate elements. Those three species were coded as ambiguous for the relevant biome-

182 region states. While it may be more accurate to describe those species as occupying

183 multiple biomes, the model that we will soon define assumes that lineages occupy only one

184 biome-region state at any given time. The biome-region character matrix and the time-

185 calibrated phylogeny for Viburnum that we used are hosted on DataDryad

186 (https://doi.org/10.5061/dryad.pvmcvdnhj).

187

188

190

191 between biomes and dispersing between regions. This process can be described in terms of

192 interactions between two fundamental subprocesses: the biome shift process and the

193 dispersal process.

194

195 types. The probability of a biome shift clearly depends on intrinsic and extrinsic factors

196 that govern how readily a lineage might adapt to the conditions in a new biome, which

197 involves a myriad of factors that we do not fully explore here. Rather, we focus specifically 
198 on modeling the effect of geographical opportunity on biome shifts (Donoghue and

199 Edwards 2014). For example, it is plausible that a species inhabiting the warm temperate

200 forests of Europe might have shifted into the tropical biome during the Early Eocene, a

201 period when tropical rain forests could be found at latitudes as extreme as $60^{\circ} \mathrm{N}$. In

202 contrast, a biome shift within Europe from a warm temperate to a tropical biome would be

203 less likely today or during any time after the global cooling trend that began with the

204 Oligocene.

The dispersal process models how lineages move between regions. The rate of

206 dispersal between regions should depend on how connected those regions are for a given

207 biome affinity. Returning to the Europe example, a tropical lineage in Southeast Asia might,

208 all else being equal, have a relatively high dispersal rate into Europe during the Early

209 Eocene, when Europe was predominantly tropical and warm temperate, as compared to

210 today, when Europe is dominated by temperate and boreal forests. This rate would,

211 however, also be influenced by geographical connectivities at the time. In this case, the

212 existence of the Paratethys Sea and the Turgai Strait may have influenced plant migrations

213 at different latitudes during the Eocene, although the fragmented northern shore of the

214 Tethys Sea facilitated the movement of tropical and subtropical plants throughout much of

215 that period (Tiffney 1985a, b; Tiffney and Manchester 2001).

216 How the biome shift and dispersal processes may behave in response to an evolving

217 biome structure is depicted in Figure 1. By characterizing known features of paleobiome

218 structure (Fig. 1A) into adjacency matrices (Fig. 1B), we can differentiate between probable

219 and improbable phylogenetic histories of biome shifts and dispersal events (Fig. 1C) based

220 on time-dependent and paleobiome-informed biome shift rates (Fig. 1D) and dispersal 
221 rates (Fig. 1E). Of the two regional biome shift histories in Figure 1C, the first history

222 invokes three events that are fully congruent with the underlying paleobiome structure.

223 The second history requires only two events, yet those events are incongruent with the

224 paleobiome structure. But which regional biome shift history is more probable? Assigning

225 probabilities to histories must depend not only on the phylogenetic placement and age of

226 the regional biome shift events, but also on the degree to which the clade evolves in a

227 paleobiome-dependent manner. We later return to how this unknown behavior of the

228 evolutionary process may be estimated from phylogenetic data, only after we define a

229 probabilistic model for the process.

An evolving spatial distribution of biomes through time

233 dynamics with a series of time-dependent graphs that are informed by the paleobiological

234 and paleogeographical literature (Fig. 2). To define our paleobiome graphs, we consulted

235 global biome reconstructions generated by Wolfe (1985), Morley (2000), Graham (2011,

236 2018), Fine and Ree (2006), Jetz and Fine (2012), and Willis and McElwain (2014) which

237 we then corroborated with biome reconstructions quantitatively estimated using the

238 BIOME4 model (Prentice et al. 1992; Kaplan et al. 2003).

239 The BIOME4 reconstructions we obtained are 1-degree global rasters that classify

240 each cell (location) as one of 27 possible biomes. BIOME4 biomes are inferred from

241 assorted botanical and climatological information (observed or imputed) that represents a

242 given time interval. For each BIOME4 reconstruction, we interpreted the 11 forest biomes

243 as approximations of the availability and connectivity of mesic forest biomes among 
244 regions, then compared those features to our literature-based reconstructions. Previously

245 published BIOME4 reconstructions were available for times corresponding to the Early-

246 Mid Eocene (Herold et al. 2014), the Late Eocene and the Oligocene (Pound and Salzmann

247 2017), the Mid/Late Miocene (Pound et al. 2011, 2012), and the Pliocene (Salzmann et al.

248 2008; Salzmann, Haywood, and Lunt 2009). For time intervals that lacked published

249 BIOME4 reconstructions, we compared our paleobiome maps to unpublished

250 reconstructions provided by P. J. Valdes (pers. comm.) that were built from proprietary

251 data. Our impression was that BIOME4 reconstructions tended to infer arid biomes that

252 were more expansive and forest biomes that were less expansive than was generally

253 supported by the paleobotanical literature. For example, the BIOME4 reconstruction

254 inferred that the Amazon contained more xeric shrubland than tropical forest at the

255 present. As such, we took presences of mesic forests under BIOME4 as conservatively

256 restricted estimates of their true distributions. That being said, the majority of literature-

257 based and BIOME4 reconstructions shared two important features: (1) the high degree of

258 availability and connectivity among tropical and warm temperate biomes in the Northern

259 Hemisphere before the Oligocene, and (2) the sudden rise of cold temperate forests

260 concomitant with the disappearance of many tropical forests, beginning with the Oligocene.

261 We classified the availability and connectivity of biomes within regions into three

262 categories of features—strong, weak, and marginal-that were appropriate to the scale of

263 the regions and the precision of the ancestral biome estimates. Biomes with a strong

264 presence displayed $\geq 25 \%$ regional coverage, biomes with a weak presence covered $<25 \%$

265 of a region, while biomes with marginal presence covered $<1 \%$ of a region. Likewise, the

266 connectivity of a biome between two regions at a given time is scored as either strong, 
267 weak, or marginal, depending on how continuously biomes are inferred to have been

268 distributed near regional adjacencies. For example, the modern distribution of the cold

269 temperate biome throughout East Asia and Europe is consistent with strong connectivity,

270 while North America is only weakly connected to cold temperate Eurasian biomes through

271 its fragmented and transient arctic connections. Independent of the distribution of biomes,

272 we similarly scored the geographical connectivity between regions as strong, weak, and

273 marginal, using the equivalent of the modern connection between Central and South

274 America through the Isthmus as Panama to minimally qualify as strong connectivity, and

275 distances between modern Europe and North America to represent weak connectivity. The

276 geographical connectivity between two regions is, generally, at least as strong as the

277 strongest biome-dependent connection for those same regions. Together, the availability

278 and connectivity for each region, each biome, and each time interval is encoded into a

279 series of paleobiome graphs, which we later use to define the rates at which biome shift

280 and dispersal events occur.

$281 \quad$ Our paleobiome graphs capture several important aspects of how mesic forest

282 biomes moved and evolved (Fig. 2). The Late Cretaceous through the Paleocene and Early

283 Eocene was a prolonged period of warm, wet conditions during which the poles had little to

284 no ice. Throughout that time, tropical forests were prevalent in all six of our regions, while

285 warm temperate forests were widespread only throughout East Asia, Europe, and North

286 America. The Eocene witnessed the emergence of a so-called boreotropical flora, which

287 contained a curious mixture (from our modern vantage point) of tropical and temperate

288 plant genera (Wolfe 1975; Tiffney 1985a, b). This largely broadleaved evergreen forest

289 type appears to have spread widely around the northern hemisphere via the Beringia Land 
290 Bridge, the North Atlantic Land Bridge, and the Tethys Sea (Tiffney 1985 a, b; Wolfe 1985;

291 Morley 2000; Willis and McElwain 2014; Graham 2011, 2018; Baskin and Baskin 2016),

292 and to have persisted through the Mid/Late Eocene. With the Oligocene, the opening of the

293 Drake Passage and the closure of the Tethys Sea redirected global ocean currents. Together

294 with steep declines in atmospheric $\mathrm{CO}_{2}$ levels, this ushered in cooler and drier conditions

295 worldwide. The ensuing global climatic changes progressively restricted tropical forests to

296 more equatorial regions, inducing the disjunction we find among modern tropical forests

297 (Latham and Ricklefs 1993; Wiens and Donoghue 2004; Donoghue 2008). As the

298 boreotropical forests receded, they were first replaced by warm temperate forests, and

299 then eventually by cold temperate and boreal forests. Following this global revolution of

300 biome structure, connectivity between Old World and New World tropical forests never

301 again matched that of the Paleocene-Eocene boreotropical beltway.

302 We illustrate how a lineage might evolve with respect to different distributions of

303 biomes within and between regions over time with the aid of Figure 2. A lineage that freely

304 disperses between regions and shifts between biomes, regardless of the historical

305 condition of the planet, might transition between regions under fully connected matrices

306 (Uninformative, first column). Lineages that are only dispersal-limited by terrestrial

307 connectivity disperse under the adjacency constraints encoded in the second column of

308 matrices (Geographical, second column). However, lineages that are dispersal-limited by

309 biome availability and connectivity might disperse according to the paleobiome patterns

310 shown in the third, fourth and fifth columns (tropical, T; warm temperate, W; and cold

311 temperate, C). For example, a lineage that is strictly adapted to the warm temperate biome

312 would disperse according to the warm temperate series of paleobiome graphs (fourth 
313 column). If that lineage shifted its affinity from a warm temperate to a tropical biome, that

314 lineage would thereafter shift between biomes and disperse between regions under the

315 adjacency matrix structures of the tropical biome (third column) until the lineage next

316 shifted biomes. However, biome shift rates also should depend on what biomes are locally

317 accessible. For example, a North American lineage would have the geographical

318 opportunity to shift from warm temperate into tropical biomes during the Paleocene, an

319 epoch when both biomes have strong presences in North America. But North American

320 tropical forests decline and then disappear throughout the Oligocene and Miocene,

321 extinguishing the opportunity for such a biome shift during more recent times. The next

322 section formalizes how we model the complex interactions between biomes, regions,

323 phylogeny, and time with these dynamics in mind.

327 interactions (if any) of its two subprocesses, the biome shift process and the dispersal

328 process. We model biome shifts using a simple continuous-time Markov chain (CTMC) with

329 time-stratified rates (i.e. piecewise constant time-heterogeneous rate matrices; Ree et al.

330 2005; Buerki et al. 2011; Bielejec et al. 2014; Landis 2017). Because transition rates

331 between regions depend in part on a lineage's biome affinity, and rates of shifting between

332 biomes depend in part on a lineage's geographical location, the two characters do not

333 evolve independently. To impose interdependence between biomes and regions, we define

334 a rate matrix over the compound state space by modifying the approach of Pagel (1994),

335 while also drawing on insights pioneered in newer trait-dependent models of discrete 
336 biogeography (Sukumaran et al. 2015; Sukumaran and Knowles 2018; Matos-Maraví et al.

337 2018; Lu et al. 2019; Klaus and Matzke 2019).

338 Accordingly, we define the CTMC to operate on the compound biome-region state,

$339(i, k)$, where $i$ is the biome and $k$ is the region. With this in mind, our goal is to compute the

340 probability of a lineage transitioning from biome $i$ in region $k$ to biome $j$ in region $l$, or

$341(i, k)$ into $(j, l)$. First, we take $\beta_{i, j}$ to model the shift rate between biomes $i$ and $j$, and $\delta_{k, l}$ to

342 model the dispersal rate between regions, $\delta_{k, l}$. Importantly, the values of $\beta$ and $\delta$

343 themselves do not directly depend on time. We eventually multiply these base rates by

344 time-dependent paleogeographical and paleoecological factors represented in our time-

345 stratified (or epoch) model.

346 Computing the transition probabilities for a time-stratified model requires that we

347 define an instantaneous rate matrix $Q(m)$ for any supported time interval, $m$. Following

348 Landis (2017), we define the rate matrix $Q(m)$ as the weighted average of several rate

349 matrices, each capturing different paleogeographical features

$$
Q(m)=w_{U} Q_{U}+w_{G} Q_{G}(m)+w_{B} Q_{B}(m)
$$

351 The three matrices on the right-hand side of Equation 1 are the (paleogeographically)

352 uninformative rate matrix, $Q_{U}$, the geographical rate matrix, $Q_{G}$, and the biome rate matrix,

$353 Q_{B}$. In reference to Figure 2, we wish to learn the relative influence of the uninformative

354 (first column), geography (second), and biome (third, fourth, or fifth) matrix features on

355 the biome shift process. Supplement 1 demonstrates how rate matrix values are computed

356 for a toy example with two regions and two biomes. 
The first rate matrix $\left(Q_{U}\right)$ may be considered as an uninformative rate matrix that sets the relative transition rates between all pairs of regions, and separately between all

359 pairs of biomes, as time-independent and context-independent,

$$
\left[Q_{U}\right]_{(i, k),(j, l)}= \begin{cases}\beta_{i, j} & \text { if biome shift }(i \neq j) \\ \delta_{k, l} & \text { if region shift }(k \neq l) \\ 0 & \text { if biome and region shift }(i \neq j \text { and } k \neq l)\end{cases}
$$

361 The effect is that biome shifts between biomes $i$ and $j$ follow the rates $\beta_{i, j}$ and dispersal

362 events follow the rates $\delta_{k, l}$ regardless of the age of a lineage or the lineage's biome-region

363 state. As we develop rate matrices for geography $\left(Q_{G}\right)$ and biomes $\left(Q_{B}\right)$ below, the second

364 role for $Q_{U}$ is that it allows for lineages to disperse or shift regardless of whether the

365 connectivity/availability of the involved regions and/or biomes are scored as strong, weak,

366 or marginal.

Because we do not know precisely what, if any, influence strong, weak, and marginal

368 features should exert upon the dispersal or biome shift processes, we estimate the relative

369 effects of those features across all adjacency matrices, the geographical adjacency matrix,

$370 A_{G}$, and the biome adjacency matrices, $A_{T}, A_{W}$, and $A_{C}$, for the tropical, warm temperate,

371 and cold temperate biomes, respectively (Fig. 2). Specifically, we assign the fixed values of

3720 and 1 to marginal and strong features, respectively, then assign all weak features one

373 shared intermediate value, estimated as $0<y<1$. In effect, y controls the degree of

374 contrast of medium-colored cells across all adjacency matrices in Figure 2. For example,

375 tropical connectivity during the Late Cretaceous is represented by the adjacency matrix

$376 A_{T}(1)$. At that time, tropical connectivity between East and Southeast Asia is strong

$377\left(\left[A_{T}(1)\right]_{\mathrm{EAs}, \mathrm{SEAs}}=1\right)$, connectivity between East Asia and Europe is weak

$378\left(\left[A_{T}(1)\right]_{\mathrm{EAs}, \mathrm{Eur}}=\mathrm{y}\right)$, and connectivity between East Asia and South America is marginal 
$379\left(\left[A_{T}(1)\right]_{\mathrm{EAs}, \mathrm{SAm}}=0\right)$. Future studies may find that one can model and reliably estimate

380 separate values of $y$ for different features, even though we do not investigate this

381 possibility at present.

382 The second rate matrix (indexed $\mathrm{G}$ for geography, $Q_{G}$ ) is structured according to

383 biome-independent paleogeographical features, such as the simple terrestrial connectivity

384 between regions. Connectivity is encoded as either as strong, weak or marginal in the

385 adjacency matrix, $A_{G}(m)$.

$$
\left[Q_{G}(m)\right]_{(i, k),(j, l)}= \begin{cases}\beta_{i, j} & \text { if biome shift }(i \neq j) \\ \delta_{k, l} \times\left[A_{G}(m)\right]_{k, l} & \text { if region shift }(k \neq l) \\ 0 & \text { if biome and region shift }(i \neq j \text { and } k \neq l)\end{cases}
$$

The third rate matrix (indexed B for biome, $Q_{B}$ ) defines the shift rates between

388 biomes and the dispersal rates between regions to depend on the spatiotemporal

389 distribution of biomes. A lineage's biome shift rate depends on whether the receiving

390 biome, $j$, has a strong, weak, or marginal presence in the region it currently occupies, $k$.

391 Likewise, the dispersal rate for a lineage that is currently adapted to biome type $i$ depends

392 on whether the source region, $k$, and destination region, $l$, share a strong, weak, or

393 marginal connection.

$$
\left[Q_{B}(m)\right]_{(i, k),(j, l)}= \begin{cases}\beta_{i, j} \times\left[A_{j}(m)\right]_{k, k} & \text { if biome shift }(i \neq j) \\ \delta_{k, l} \times\left[A_{j}(m)\right]_{k, l} & \text { if region shift }(k \neq l) \\ 0 & \text { if biome and region shift }(i \neq j \text { and } k \neq l)\end{cases}
$$

It is crucial to recognize that $Q_{B}(m)$ defines shift rates involving biome $j$ to depend

396 on the adjacency matrix for biome $j$ during time interval $m$. This key property means that

397 lineages currently adapted to biome $j$ disperse with rates according to the interregional 
connectivity of biome $j$, and lineages newly adapting to biome $j$ do so at a rate depending on the local availability of biome $j$.

The transition rates (and probabilities) between biome-region pairs are not

401 expected to be symmetric or equal across time intervals. For example, if biome $j$ first

402 appears in region $k$ during time interval $m+1$, then we would potentially see an increase

403 in the biome shift rate into biome $j$, i.e. $[Q(m)]_{(i, k),(j, k)}<[Q(m+1)]_{(i, k),(j, k)}$. Nor are

404 transition rates necessarily symmetric or equal within a given time interval. If region $k$

405 contains biome $i$ during time interval $m$, but region $l$ does not, then we might find that

406 lineages adapted to biome $i$ disperse less easily from $k$ into $l$ than $l$ into $k$, i.e.

$407[Q(m)]_{(i, k),(i, l)}<[Q(m)]_{(i, l),(i, k)}$. Similarly, if region $k$ contains biome $i$ but not biome $j$

408 during time interval $m$, then lineages inhabiting region $k$ might tend to shift less easily from

409 biome $i$ into $j$ than from $j$ into $i$, i.e. $[Q(m)]_{(i, k),(j, k)}<[Q(m)]_{(j, k),(i, k)}$.

$410 \quad$ Fluctuating asymmetries in the rates over time means that each biome-region state

411 may exhibit different source-sink dynamics across that timescale. During a period of low

412 accessibility, a biome-region state might rebuff immigrants and lose occupants when acting

413 as a source, but then gain and retain inhabitants during a later phase should that biome-

414 region become a local refugium when acting as a sink (Goldberg et al. 2005). These

415 fluctuating source-sink dynamics may be characterized by the stationary distribution,

416 which defines the expected proportion of lineages found in each biome-region state

417 assuming lineages evolve along an infinitely long branch within a given time interval.

418 Biome-regions that are easy to enter and difficult to leave tend towards higher stationary

419 probabilities for a given time interval. 
We approximate the stationary probability for biome $i$ in region $k$ during time

$421 \quad$ interval $m$ with

$$
\pi(m)_{(i, k)}=\left[e^{\mu Q(m)}\right]_{1,(i, k)}
$$

423 where $\mu$ is a rate taken to be sufficiently large that the evolutionary process reaches

424 stationarity. Note that the bracketed term on the righthand side of the equation is the

425 transition probability matrix for changes between biome-region pairs. In theory, when $\mu$ is

426 large, all rows in this matrix have arbitrarily similar transition probabilities, which lets us

427 take any row (e.g. the first row) to represent the stationary probabilities.

428 The time-dependent source-sink dynamics in Figure 3 show how the availability of

429 and connectivity between regional biomes structure each time interval's stationary

430 distribution. Stationary probabilities before the Oligocene tend to favor tropical biomes in

431 all regions but favor cold temperate biomes afterwards. This means that if the historical

432 spatial structure of biomes is relevant to biogeography, then lineages originating in the

433 Paleogene would more likely be adapted to tropical than to cold temperate forests simply

434 because cold temperate forests were a more marginal biome during that period of Earth's

435 history.

436 We can now completely define the time stratified rate matrix, $Q(m)$, and the

437 stationary frequencies at the root of a phylogeny, $\pi\left(m_{\text {root }}\right)$, where $m_{\text {root }}$ is the time

438 interval corresponding to the root node age. Together, these model components let us

439 compute the probabilities of lineages transitioning from one biome-region pair to another

440 while accounting for the spatiotemporal dynamics of biomes, and thus compute the

441 phylogenetic model likelihood with the discrete state pruning algorithm (Felsenstein

442 1981). 
Now that we have fully defined the model, there are several implicit properties that

444 are worth stating explicitly. First, a lineage cannot both shift its biome affinity and disperse

445 into a new region in the same moment of time; one event is needed for each transition, and

446 so event order matters. While we think this assumption is reasonable in many cases,

447 including in Viburnum, it is certainly conceivable that region and biome shifts could occur

448 simultaneously. We elaborate on this point in the Discussion, but note here that it would be

449 possible to explore the effect of modeling such paired transitions by adapting

450 parameterizations designed for multiple-nucleotide substitution codon models (Koisol et

451 al. 2007). Second, the relative importance of the matrix feature weights $\left(w_{U}, w_{G}, w_{B}\right)$ and of

452 the weak availability/connectivity weight (y) are estimated from the data: the matrix $Q(m)$

453 reduces to the uninformative matrix, $Q_{U}$, when $w_{U}=1$, while the importance of the

454 historical structure of biomes is most pronounced when $w_{B}$ is large compared to other $w$

455 and $y$ parameters. Third, the process models lineages as being predominantly present in a

456 single region and biome at a time without influencing speciation or global extinction rates,

457 which are assumptions we made both to simplify the exposition of the method, but also to

458 reduce computational burden. The Discussion brings more attention to all of these

459 properties.

460

Bayesian inference

463 (MCMC) algorithm implemented in RevBayes (Höhna et al. 2016). RevBayes scripts and

464 datasets are available at http://github.com/mlandis/biome_shift. The first 10\% of

465 posterior samples were discarded as burn-in. All parameter estimates have effective 
466 sample sizes well over 200. Two independent chains were run per analysis to verify MCMC

467 convergence. We analyzed our data under three model settings: the Paleobiome setting that

468 used the time-heterogeneous graphical structure presented in Figure 2; the Modern Biome

469 setting that used the graphical structure from the Recent time interval to represent all time

470 intervals; and the Null Biome setting that ignored regional and biome structure by fixing

$471 \quad w_{U}=1$.

472

Departing from the general model description above, we reparameterized our

473 applied model to eliminate informative priors wherever possible. This helped ensure that

474 our posterior estimates are driven by the data through the likelihood function, not the

475 prior (discussed further in Supplement 2). We assigned uninformative prior distributions

476 to our graph weights, $\left(w_{U}, w_{L}, w_{B}\right) \sim \operatorname{Dirichlet}(1,1,1)$, and to our weak feature strength

477 parameter, $\mathrm{y} \sim$ Uniform $(0,1)$. We treated each biome shift rate as an independently

478 estimated parameter, $\beta_{i, j} \sim \operatorname{Uniform}(0,1)$, although we fixed the biome shift rates between

479 tropical and cold temperate biomes equal to zero. Fixing those transition rates to zero both

480 reduces the number of free parameters and reflects the observation that, analogous to the

481 geographical structure of regions, biomes have an important ecological and climatological

482 structure (Whittaker 1970). In Viburnum, this assumption is reasonable, as the clade

483 contains no tropical-cold temperate sister species pairs, and previous ancestral biome

484 estimates for the group did not confidently infer any shifts between those biomes (Landis

485 et al. 2020). Because we constrained biome-independent dispersal between regions

486 through graphical structures $\left(Q_{G}\right)$ and weight parameters $\left(w_{U}\right.$ and $\left.w_{G}\right)$, we fixed the

487 relative dispersal rate to $\delta_{k, l}=1$ (which is potentially rescaled by $Q_{G}$ and $w_{G}$ ). Thus, the

488 relative biome shift rates $\beta$ and dispersal rates $\delta$ all have values between 0 and 1 . To model 
489 the relative proportion of biome shifts to dispersal events, we multiply $\beta$ by the factor $f_{\beta} \sim$

490 Uniform $(0,1)$ and multiply $\delta$ by $f_{\delta}=\left(1-f_{\beta}\right)$. Finally, we rescaled the instantaneous rate

491 matrix, $Q$, for the entire evolutionary process by a global clock parameter, $\mu \sim$

492 LogUniform $\left(10^{-4}, 10^{1}\right)$, that is uniformly distributed over orders of magnitude. For the

493 Viburnum analysis, we used Bayes factors (Jeffreys 1935) to compare the relative fit of the

494 Paleobiome, Modern Biome, and Null Biome models to the data. Power-posterior

495 distributions were approximated with a parallelized MCMC sampler (Höhna et al. 2017).

496 Marginal likelihoods were estimated from those power-posterior samples using the

497 stepping stone algorithm (Xie et al. 2011).

$498 \quad$ Our analysis produced several inferences, which we summarized in various ways.

499 Ancestral states and stochastic mappings (Huelsenbeck et al. 2003) were generated during

500 MCMC sampling by first drawing ancestral state estimates for all nodes in phylogeny, then

501 sampling evolutionary histories using a uniformization method (Rodrigue et al. 2008) that

502 was adapted for time-stratified models (Landis et al. 2017). Ancestral state estimates show

503 the posterior probabilities for biome-region states for each node. Lineage-state proportions

504 through time were computed from the posterior distributions of stochastically mapped

505 histories. We computed the posterior mean count of lineage-states through time as the

506 number of lineages in each state for each time bin across posterior samples divided by the

507 total number of posterior samples. Lineage-state counts were converted into lineage-state

508 proportions by dividing each count by the total number of lineages in that time bin to give

509 proportions that lie between 0 and 1 . In addition, we classified whether or not each

510 lineage-state for each time bin was congruent with any locally prominent biome as defined

511 by the paleobiome graph (Fig. 2). Each binned state was labeled as a biome mismatch if the 
512 lineage's biome had only a marginal presence in the lineage's region. Otherwise, the state

513 was labelled as a biome match. To summarize these results, we also computed the

514 proportion of tree length where lineage states match or mismatch paleobiome structure in

515 three ways: for the total tree length, for tree length before the Oligocene ( $>34 \mathrm{Ma})$, and for

516 tree length after the Oligocene ( $\leq 34 \mathrm{Ma}$ ). To assess whether the use of the Paleobiome

517 model unduly biased posterior estimates (e.g. by inflating the prior expectations of biome

518 match proportions), we generated stochastic mappings conditional on the phylogeny and

519 tip states while sampling model parameters from the prior (details given in Supplement 2).

520 We were also interested in the ordered event series that resulted in major

521 transitions between biomes and regions. For biomes $A, B$, and $C$ and regions $X, Y$, and $Z$, we

522 named the six series patterns for pairs of events. Series in which species shift biomes and

523 then disperse $(A X \rightarrow B X \rightarrow B Y)$ are called biome-first event series. In contrast, region-first

524 series have dispersal followed by a biome shift event $(A X \rightarrow A Y \rightarrow B Y)$. The remaining four

525 event series involve two consecutive biome shift or two dispersal events. Biome reversal

$526(A X \rightarrow B X \rightarrow A X)$ and region reversal $(A X \rightarrow A Y \rightarrow A X)$ sequences indicate event series in

527 which the lineage departs from and then returns to its initial state $(A X)$. Analogously, biome

528 flight $(A X \rightarrow B X \rightarrow C X)$ and region flight $(A X \rightarrow A Y \rightarrow A Z)$ sequences are recognized by

529 series of two biome shifts or two dispersal events that leave the lineage in a new state $(C X$

530 or $A Z$ ) relative to the lineage's initial state $(A X)$. We computed the proportion of each

531 series type for a single posterior sample by classifying stochastically mapped state triplets

532 (event series of length two) in our phylogenetic tree using a simple root-to-tip recursion.

533 To start the recursion, we took the stochastically mapped root state to be the second state 
534 in the triplet, $X_{\text {root }}$, then sampling the preceding state, $X_{\text {subroot }}$, from the sampling

535 distribution obtained by Bayes rule

$536 P\left(X_{\text {subroot }}=(i, k) \mid X_{\text {root }}=(j, l), Q\left(m_{\text {root }}\right)\right) \propto \frac{\left[Q\left(m_{\text {root }}\right)\right]_{(i, k),(j, l)}}{\sum_{(x, y) \neq(i, k)}\left[Q\left(m_{\text {root }}\right)\right]_{(i, k),(x, y)}} \times \frac{\left[\pi\left(m_{\text {root }}\right)\right]_{(i, k)}}{\left[\pi\left(m_{\text {root }}\right)\right]_{(j, l)}}$

537 where $Q\left(m_{\text {root }}\right)$ is the root node's rate matrix and $\pi\left(m_{\text {root }}\right)$ is its stationary distribution

538 with values determined by the evaluated posterior sample. Following that, we traversed

539 towards the tips of the tree to collect changes in the stochastic mapping for each lineage's

540 biome-region state, classifying the state triplet's type, and updating the triplet states

541 appropriately (i.e. the new second and third states replace the old first and second states)

542 with each step of the recursion.

543 Finally, we wished to examine if and how the distribution of evolutionary events

544 and event series changed with time under alternative assumptions about the biome

545 structure. We were particularly interested in two classes of event proportions: proportions

546 of various types of biome shift and dispersal events, and proportions of the various types of

547 event series. To estimate the posterior proportions of biome shift and dispersal event types

548 through time, for each posterior sample, we divided the count for each distinct biome shift

549 and dispersal event type by the total number of events within each major time interval.

550 Event proportions from the Late Cretaceous could not be reliably estimated, as the two

551 Viburnum lineages that existed between the origin of the group ( 70 Ma) and the end of

552 the Late Cretaceous ( $\sim 66 \mathrm{Ma}$ ) rarely changed in biome-region state. Although we

553 normalized our event proportions using all 126 distinct dispersal and biome shift event

554 types, our results only display the four biome shift and four dispersal event types among all

555 combinations of the warm and cold temperate forests of East Asia and North America. In a 
556 similar manner, we computed the posterior proportions for all six types of event series,

557 using the time of the second event in each series for each series age.

558 We provide the posterior means and the $80 \%$ and $95 \%$ highest posterior densities

559 (HPD80 and HPD95, respectively) for each time interval, event or event series type, and

560 biome structure model. Constructing Bayesian credible intervals for these proportions is

561 somewhat unusual, since the proportions are ratios of counts (i.e. not all non-negative real

562 numbers are supported). As a result, several HPDs share the exact same bounds,

563 particularly when the total number of events is low (e.g. values such as $1 / 2$ or $1 / 3$ ).

Simulation experiment

566 We measured how reliably we can select models in which biome structure

567 influences the biome shift process $\left(w_{B}>0\right)$ for Viburnum with simulated data. All

568 simulations assumed the same Viburnum phylogeny used in the empirical example and

569 used the same biome and regions designated by the paleobiome structure model. We

570 simulated data under five conditions that primarily adjusted the relative weight for $w_{B}$,

571 named: null effect, where $\left(w_{U}, w_{G}, w_{B}\right)=(1,0,0)$; weak effect, where $\left(w_{U}, w_{G}, w_{B}\right)=$

$572(1,2,4) / 7$; medium effect, where $\left(w_{U}, w_{G}, w_{B}\right)=(1,2,8) / 11$; strong effect, where

$573\left(w_{U}, w_{G}, w_{B}\right)=(1,2,16) / 19 ;$ and very strong effect, where $\left(w_{U}, w_{G}, w_{B}\right)=(1,2,32) / 35$; with

574 each denominator ensuring the weights sum to 1 . For all conditions, we assumed $f_{\beta}=0.75$,

$575 f_{\delta}=0.25$, and $y=0.1$. Biome shift rates were set to equal 1 , except transitions between

576 cold temperate and tropical forests, which were set to 0 . The event clock was set to $\mu=$

5770.03 , except for the null condition, which was assigned a slower rate of $\mu=0.01$ to account

578 for the fact that fewer event rate penalties are applied to it than the non-null conditions. 
579 We then simulated 100 replicate datasets in RevBayes for each of the four conditions under

580 the regional biome-shift model described above, and estimated the posterior density for

581 each simulated dataset using MCMC in RevBayes.

We were primarily concerned with how our posterior estimates of $w_{B}$ respond to

583 differing simulated values for $w_{B}$. To summarize this, we first report the posterior median

584 values of $w_{B}$ across replicates so they may be compared to the true simulating value. Next,

585 we computed what proportion of our replicates select a complex model allowing $w_{B}>0$ in

586 favor of a simpler model where $w_{B}=0$ using Bayes factors. Bayes factors were computed

587 using the Savage-Dickey ratio (Verdinelli and Wasserman 1995), defined as the ratio of the

588 prior probability divided by the posterior probability, evaluated at the point where the

589 complex model collapses into the simpler model (i.e. $w_{B}=0$, in our case). We

590 approximated the posterior probability at this collapse-point by smoothing our posterior

591 samples for $w_{B}$ with a beta-kernel density estimator (Moss and Tveten, 2019). We interpret

592 the strength of significance for Bayes factors as proposed by Jeffreys (1961), requiring at

593 least 'Substantial' support $(\mathrm{BF}>3)$ to select the more complex model $\left(w_{B}>0\right)$.

RESULTS

599 datasets simulated under the Null condition $\left(w_{B}=0\right)$ signalled Substantial support (or

600 greater) for the paleobiome-dependent model $\left(w_{B}>0\right)$, indicating a low false positive rate.

601 Only $9 \%$ of datasets simulated under Weak effects $\left(w_{B}=4 / 7 \approx 0.57\right)$ generated No 
602 support for the $w_{B}>0$ model, while $\sim 32 \%$ of those replicates qualified as Substantial

603 support or greater. Data simulated under the Moderate condition $\left(w_{B}=8 / 11 \approx 0.73\right)$

604 rejected the simple model 57\% of the time with at least Substantial support. Under Strong

$605\left(w_{B}=16 / 19 \approx 0.84\right)$ simulation conditions, we selected models where $w_{B}>0$ in $81 \%$ of

606 cases, with Strong support in $65 \%$ of cases. Data simulated under Very Strong effects $\left(w_{B}=\right.$

$60732 / 35 \approx 0.91$ ) generated support for models with $w_{B}>088 \%$ of the time, with over half

608 of all replicates (54\%) drawing Very Strong or Decisive support. Coverage frequency

609 among simulations was consistently high across conditions, but with fairly wide credible

610 intervals corresponding to the highest 95\% posterior density (Fig. 4A). Because the

611 posterior probability of $w_{B}=0$ is used to approximate Bayes factor ratios, their

612 relationship is made apparent by noting that the density of HPD95 lower bound estimates

613 close to the value $w_{B}=0$ (Fig. $4 \mathrm{~A}$ ) is correlated with the proportion of simulations that

614 award no support to the $w_{B}>0$ model (Fig. 4B).

618 model $(p>0.99)$, no model reconstructed a single ancestral biome affinity with probability

619 greater than $p>0.95$ (Fig. 5). Where the Paleobiome analysis inferred East Asian biome

620 affinities that favored a warm temperate $(p=0.88)$ or tropical $(p=0.09)$ but not a cold

621 temperate $(p=0.03)$ origin, the Modern Biome analysis favored a cold temperate $(p=$

622 0.67) then warm temperate $(p=0.31)$ origin for Viburnum while assigning negligible

623 probability to a tropical origin $(p=0.01)$. Relative to the Paleobiome estimates, the Null

624 Biome analysis also assigned higher probabilities towards colder biomes (warm, $p=0.52$; 
625 cold, $p=0.45$; tropical, $p=0.02$ ). Early diverging Viburnum lineages tended to follow

626 warm/tropical biome affinities under the Paleobiome analysis or the cold/warm affinities

627 under the Modern/Null Biome analyses before the Oligocene (>34 Ma). During the

628 Oligocene (34-22 Ma), when cold temperate forests first expanded, many nodes still

629 retained the warmer (Paleobiome) or colder (Modern Biome) affinities characteristic of the

630 corresponding biome structure model, such as the most recent common ancestor (MRCA)

631 of $V$. reticulatum and V. ellipticum or the MRCA of $V$. rufidulum and $V$. cassinoides. Otherwise,

632 most ancestral biome inferences were consistent across the three models, beginning with

633 the Mid/Late Miocene $(<16 \mathrm{Ma})$.

634 The three biome structure models recovered different proportions of ancestral

635 lineage-states through time, particularly before the Mid/Late Miocene (>16 Ma; Fig. 6A-C).

636 Between the Paleocene and the Early Miocene, tropical lineages in East Asia and Southeast

637 Asia constituted $>20 \%$ diversity, declining to $\sim 12 \%$ of modern diversity under the

638 Paleobiome analysis. Cold temperate lineages were nearly absent until the end of the

639 Oligocene (34 Ma), but steadily rose to constitute roughly $25 \%$ of diversity by the

640 Early/Mid Miocene (ca. 20 Ma). By comparison, Modern Biome estimates enriched the

641 proportion of cold temperate viburnums, while reducing support for warm temperate and

642 nearly eliminating support for tropical origins; tropical lineages remained in comparatively

643 low proportion until the Miocene (<22 Ma). The Null Biome analysis estimated

644 proportions of warm and cold temperate lineages similar to those of the Modern Biome

645 analysis from the Late Cretaceous (100 Ma) until the Oligocene (34 Ma), but with more

646 Southeast Asian warm temperate lineages throughout. 
For what proportion of time did lineages have biome affinities that were congruent

648 with locally accessible biomes? Biomes rarely mismatched between lineages and regions

649 under the Paleobiome setting (1.1\% of tree length), with the mismatches increasing under

650 the Modern Biome (8.6\%) and Null Biome (8.7\%) settings. Lineages were most often

651 mismatched with their regions' biomes before the Oligocene (Figs. 6D-F), where the pre-

652 Oligocene proportion of mismatched branch lengths was always higher (Paleobiome, 5.8\%;

653 Modern Biome, 52.6\%; Null Biome, 47.1\%) than the post-Oligocene proportion (Paleobiome,

654 0.3\%; Modern Biome, 0.8\%; Null Biome, 1.7\%) or the treewide proportions (above). It is

655 unlikely that our posterior inferences are artifacts caused by undesirable interactions

656 between our prior model and our biome structure models, since the prior-like distributions

657 of stochastic mappings that we generated under the Paleobiome, Modern Biome, and Null

658 Biome settings were nearly indistinguishable from one another (Supplement 2; Fig. S1).

659 To illuminate why the Paleobiome analysis produces distinctly warmer ancestral

660 biome estimates, we turn to the fitted stationary probability for the root state, $\pi\left(m_{\text {root }}\right)$,

661 (Fig. 7). Within East Asia, root node stationary probabilities estimated under the

662 Paleobiome setting favored warm temperate or tropical forests over cold temperate forests

$663\left(\pi_{\mathrm{Trop}+\mathrm{EAs}}=0.06, \pi_{\mathrm{Warm}+\mathrm{EAs}}=0.10, \pi_{\mathrm{Cold}+\mathrm{EAs}}=0.02\right)$. The Modern Biome stationary

664 probabilities instead favored cold or warm temperate forests over tropical forests

$665\left(\pi_{\mathrm{Trop}+\mathrm{EAs}}=0.03, \pi_{\mathrm{Warm}+\mathrm{EAs}}=0.07, \pi_{\mathrm{Cold}+\mathrm{EAs}}=0.08\right)$. Like the Modern Biome analysis,

666 stationary probabilities under the Null Biome setting tended towards cold or warm

667 temperate forests $\left(\pi_{\mathrm{Trop}+\mathrm{EAs}}=0.04, \pi_{\mathrm{Warm}+\mathrm{EAs}}=0.06, \pi_{\mathrm{Cold}+\mathrm{EAs}}=0.06\right)$, noting that the

668 stationary probability per biome is uniform across regions by the design of the model.

669 While the posterior root node stationary probabilities varied across biome-regions 
670 depending on what biome structure model was assumed (Fig. 7), all corresponding prior

671 probabilities are approximately equal (Supplement 2; Fig. S2), suggesting that any

672 differences in the posterior estimates are driven by the data through the likelihood

673 function, and not forced through an induced prior.

674 Despite such differences between the Paleobiome and Modern Biome analyses in

675 their ancestral state estimates and stationary probabilities, their parameter estimates for

676 the base rate of change $(\mu)$, the proportion of biome shifts $\left(f_{\beta}\right)$ to dispersal events $\left(f_{\delta}\right)$, and

677 the graph weights $\left(w_{U}, w_{G}, w_{B}\right)$ were remarkably similar (Table 1$)$. Both biome structure

678 models estimate posterior means for $w_{B}$ greater than 0.91 ; i.e., stronger in effect than

679 assumed under the Very Strong simulation scenario (Fig. 4). Both models estimated

680 credible intervals for $w_{B}$ with lower bounds greater than 0.75 and posterior probabilities of

$681 w_{B}=0$ that were indistinguishable from zero, each corresponding to Decisive support for

682 their respective biome structure models. More accurate Bayes factors estimated using

683 marginal likelihoods unequivocally selected either the Paleobiome $\left(\mathrm{BF}_{\mathrm{PN}}>10^{6}\right)$ and the

684 Modern Biome $\left(\mathrm{BF}_{\mathrm{MN}}>10^{6}\right)$ over the Null Biome structures for modeling biome shifts in

685 Viburnum, but found only mild support in favor of the Paleobiome over the Modern Biome

686 structure $\left(\mathrm{BF}_{\mathrm{PM}} \approx 1.8\right)$. Because inference under the Null Biome model set $\mathrm{y}=1$, posterior

687 estimates of $\left(w_{U}, w_{G}, w_{B}\right)$ are indistinguishable from the prior. Parameter estimates for the

688 relative biome shift rates differed across the three biome structure models, however. The

689 Paleobiome estimates have relatively low cold-into-warm and high tropical-into-warm

690 biome shift rates (posterior medians of $\beta_{T W}=0.67, \beta_{W T}=0.29, \beta_{W C}=0.81, \beta_{C W}=0.38$ )

691 when compared to the Modern Biome $\left(\beta_{T W}=0.50, \beta_{W T}=0.39, \beta_{W C}=0.81, \beta_{C W}=0.65\right)$

692 and the Null Biome estimates $\left(\beta_{T W}=0.54, \beta_{W T}=0.31, \beta_{W C}=0.74, \beta_{C W}=0.71\right)$. 
Finally, we found that the Paleobiome analysis estimated proportions of biome shift

694 and dispersal events that are more temporally dynamic than those proportions estimated

695 under the Modern Biome and Null Biome models (Fig. 8A-C). Under the Paleobiome

696 estimates, dispersal events from East Asia into North America within the warm temperate

697 biome were relatively common throughout the Late Eocene. With the onset of Oligocene

698 cooling, biome shifts from warm into cold temperate forests in East Asia rose from low to

699 high proportions to become the most frequent transition type. In contrast, event

700 proportions under the Modern Biome and Null Biome analyses reconstructed high

701 proportions of biome shifts between the warm and cold temperate forests of East Asia, ever

702 since Viburnum first originated in the Late Cretaceous through the present. Paleocene

703 dispersal of cold temperate lineages from East Asia into North America was also found to

704 be relatively common when compared to the Paleobiome reconstruction. Regarding the

705 event series proportions, biome reversal, biome-first, and region-first series were generally

706 more common than biome flight, region flight, and region reversal series (Fig. 8D-F). The

707 biome reversal event series was the most common event series type across all time

708 intervals under the Modern Biome and Null Biome analyses, but not under the Paleobiome

709 analysis. With the Paleobiome model, we found that the proportion of biome reversal series

710 was lower, and the proportion of region-first series was higher, when compared to the

711 other biome structure analyses, together creating a time interval between the Late Eocene

712 and the Middle Miocene during which region-first events outpaced all other types of series. 
The probability that a lineage will shift into a new biome is determined in part by

716 geographical opportunities. Because the availability and connectivity of biomes varies

717 across regions, evolutionary lineages do not share the same geographical opportunities to

718 adapt to new biomes. Moreover, those geographical opportunities have changed as the

719 spatial structure of Earth's biomes evolved over time. As an evolutionary inference

720 problem, the temporal dynamics of geographical opportunity is concerning: we typically

721 infer ancestral biomes based on the phylogenetic distribution of biomes from extant

722 species, yet their ancestors were likely exposed to geographical opportunities that were

723 significantly (perhaps even radically) different from the opportunities of their living

724 descendants.

725 Here, we have developed a Bayesian framework to model how phylogenetic lineages

726 gain affinities with new biomes and disperse between regions in a manner reflecting the

727 historical configuration of biomes through space and time. To do so, we modeled a time-

728 stratified regional biome shift process using continuous-time Markov chains. The model is

729 parameterized to allow biome shift and dispersal rates to depend on empirically structured

730 paleobiome graphs, where each graph describes the availability and connectivity of biomes

731 among regions within a given time stratum. We conducted a simple simulation experiment

732 to show that we can identify which comparative datasets were shaped by paleobiome

733 structure $\left(w_{B}>0\right)$ using Bayes factors, provided the strength of the effect was at least

734 moderately strong, even though $w_{B}$ is difficult to estimate precisely (Fig. 4). We then fitted

735 our new model to estimate ancestral biomes and regions for Viburnum. In discussing our

736 results, we focus on two principal aspects of our study: first, an interpretation of our

737 empirical findings in Viburnum and how these may inform other studies seeking to 
738 estimate ancestral biomes or regions; and, second, an examination of the model's

739 assumptions and properties, and how the model's realism may be improved in future work.

Viburnum first diversified in the Paleocene and Eocene (66-34Ma), a period when

743 boreotropical forests dominated and connected the northern continents (Wolfe 1985;

744 Graham 2011; Willis and McElwain 2014). Cold temperate forests that experienced long

745 freezing periods were globally rare until after the Oligocene $(<34 \mathrm{Ma})$. Although we

746 inferred an East Asian origin regardless of what biome structure model was assumed,

747 ancestral biome estimates under the three structure models differed in important ways. In

748 the Paleobiome analysis, the ancestral biome of the crown node was probably warm

749 temperate $(p=0.88)$ and possibly tropical $(p=0.09)$, and a cold temperate origin could

750 decisively be ruled out ( $p<0.05$; Fig. 5A). When we assumed that biome structure had

751 always resembled today's structure (Modern Biome), the crown node support changed,

752 instead favoring a cold temperate $(p=0.67)$ or possibly a warm temperate $(p=0.31)$

753 origin (Fig. 5B). The Null Biome reconstruction also recovered a warm $(p=0.52)$ or cold

$754(p=0.45)$ temperate origin, despite the fact that the Null Biome inference assumed that all

755 biomes are present in all regions at all times (Fig. 5C). Mismatches between lineage biome

756 affinities and regionally available biomes were highest among pre-Oligocene lineages $(>34$

757 Ma). Though cold temperate lineages remained in low proportions $(\sim 5 \%)$ until the

758 Oligocene under the Paleobiome analysis (Fig. 6A), the Modern/Null Biome analyses

759 maintained high proportions of cold temperate lineages in East Asia ( $>30 \%)$ and North

760 America (7\%) in the Eocene (Fig. 6B,C). Over 53\% and 47\% of pre-Oligocene branches bore 
761 mismatched biomes under the Modern and Null Biome analyses, respectively, but only $6 \%$

762 of those branch lengths were mismatched with biomes under the Paleobiome model (Fig.

763 6D-F). Because of the global rarity of the cold temperate biome during the period of early

764 Viburnum evolution, we favored the warm temperate or tropical origin of Viburnum under

765 the Paleobiome analysis.

766 Yet, despite stark differences in the Paleobiome and Modern Biome models,

767 parameter estimates under both conditions found the spatial distribution of biomes to be

768 the primary factor in explaining how viburnums came to live where they do today $\left(w_{B}>\right.$

769 0.92, i.e. compatible with the Very Strong condition used in the simulation experiment).

770 Because the ability to estimate ancestral states or to fit evolutionary parameters decays as

771 the evolutionary timescale deepens, we expect that both the Paleobiome and Modern Biome

772 analyses primarily fit their parameters to phylogenetic patterns of variation pronounced at

773 the shallowest timescales. True to this, model selection by Bayes factors only slightly

774 prefers the Paleobiome over the Modern Biome structure. All else being equal, however,

775 older Viburnum lineages should disperse and biome shift in a manner that is similarly

776 limited by geographical opportunities. The static geographical opportunities assumed

777 under the Modern Biome structure induced stationary probabilities that project today's

778 colder conditions back into the Late Cretaceous, while the dynamic Paleobiome structure

779 favored hotter conditions unlike those at present (Figs. 2 and 7). The lesson we take from

780 this is that inferring the fundamental behavior of the process is not always sufficient for

781 estimating ancestral states; inferring if and how that behavior responds to changing

782 historical conditions is also necessary. 
We note that an East Asian origin in warm temperate or tropical forests is consistent

784 with several other relevant lines of evidence developed in the study of Viburnum evolution,

785 biogeography, and ecology. Previous efforts to reconstruct the ancestral biome of Viburnum

786 have weakly favored warm temperate (Spriggs et al. 2015) or cold temperate (Lens et al.

787 2016) conditions; neither study definitively supported or ruled out a cold temperate origin.

788 Similarly, Edwards et al. (2017) established a relationship between cold temperate

789 conditions and the evolution of deciduousness in Viburnum, but could not resolve whether

790 the MRCA was deciduous (cold-adapted) or evergreen (tropical or warm-adapted). Landis

791 et al. (2020) estimated a warm temperate origin of Viburnum, with no support for a cold

792 temperate origin, through a combined-evidence tip-dating analysis (Ronquist et al. 2012)

793 that included fossil pollen coded with biome characters to inform the ancestral biome

794 estimates. As a fossil-based estimate, the finding of a non-freezing origin of Viburnum

795 cannot be accepted unconditionally; the estimate depends crucially upon the accuracy of

796 biome state assignments to the fossil taxa, and also upon the spatial and temporal biases

797 inherent to fossil deposition and recovery. But, importantly, the fossil-aware biome

798 estimates of Landis et al. (2020) were obtained under the equivalent of our Null Biome

799 model, while the fossil-naive estimates in the present study were obtained under the

800 Paleobiome model. It is highly satisfying that both studies rule out a cold temperate

801 ancestry for Viburnum, and that they do so by leveraging alternative lines of paleobiological

802 evidence: the phylogenetic placement of fossils assigned to particular biomes in one case,

803 and the inferred spatial distribution of biomes through time in the other.

804 Examining only extant Viburnum species, the clade displays considerable variation

805 in both which biomes and which regions lineages occupy. Yet, each region does not contain 
806 equal proportions of lineages with affinities to the three biomes. There are several possible

807 causes for this imbalance. In many cases, lineages may simply inhabit regions that lack

808 certain biomes; it is not surprising that there are no extant tropical lineages in North

809 America given that tropical forests have been marginal there since the Oligocene. In other

810 cases, lineages may not have had long enough periods of time for certain biome shifts. For

811 example, all neotropical lineages are adapted to warm temperate (cloud) forests, yet none

812 of them have adapted to the adjacent tropical forest biome. Given the young age of the

813 neotropical radiation, it is possible that there has not been enough time for them to shift

814 into the accessible tropical forests. In this case we can imagine that biological factors (e.g.,

815 interactions with other species-competitors, herbivores, etc.-that have long occupied

816 tropical forests) may have played a significant role in limiting this shift (Donoghue and

817 Edwards 2014). In other cases, the imbalance may concern differential rates of speciation

818 or extinction within biomes. For instance, there are relatively few tropical Viburnum

819 species given the age and region of origin for the clade and given the age of Asian tropical

820 biomes. If tropical viburnums experienced increased extinction rates (or decreased

821 speciation rates) as they remained in an older biome, that effect would give rise to a

822 pattern of scattered, singular, distantly related, and anciently diverging tropical lineages

823 (depauperons of Donoghue and Sanderson 2015). This is precisely what we see in the case

824 of V. clemensiae, V. amplificatum, and V. punctatum (Spriggs et al. 2015). From analyses

825 under our simple Paleobiome model, it appears that temporal, geographical, and ecological

826 influences on rates of character evolution and lineage diversification may all be important

827 factors in explaining why Viburnum is distributed as it is across regions and biomes. 
829 of events (e.g., trait-first versus climate-first in the evolution of cold tolerance; Edwards et

830 al. 2015), we nevertheless explored how incorporating information on the past distribution

831 of biomes might influence the inference of biome-first versus region-first event series.

832 Specifically, we asked whether Viburnum lineages tended to shift biomes first or disperse to

833 a new region first when radiating through the mesic forests of Eurasia and the New World.

834 Taking the mean proportions across time intervals, we found that when Viburnum lineages

835 both disperse into new regions and shift into new biomes, region-first event series (28\% of

836 series) are more common than biome-first (19\%) series under the Paleobiome model.

837 Alone, this result is difficult to interpret, since the relative number and size of biome and

838 region states will influence what constitutes a biome shift or dispersal event. Using the

839 Modern analysis as a point of reference, we find a comparatively neutral relationship, with

840 roughly equal proportions of biome-first (20\%) and region-first (21\%) series, while under

841 the Null Biome analysis the Paleobiome relationship is inverted (biome-first, 22\%; region-

842 first, 19\%). When all regions contain all biomes (Null Biome), it makes sense that the ratio

843 of biome-first to region-first series is highest, and that it decreases when the distribution of

844 biomes is not uniform across regions (Paleobiome and Modern Biome). In the case of

845 Viburnum, it appears that several key regional shifts between Eastern Asia and North

846 America occurred a relatively long time ago, when northern latitudes were still primarily

847 covered by warm temperate forests (Fig. 8A). The biome shifts into cold temperate forests

848 occurred later, as cooling climates spread across communities that were already

849 assembled, which is compatible with the 'lock-step' hypothesis of Edwards et al. (2017).

850 Consistent with this scenario, we found that region-first event series do not become the 
851 most common series type (over 35\%) until the Late Oligocene under the Paleobiome model

852 (Fig. 8D). Such region-first event series have also been inferred in several recent analyses,

853 most notably by Gagnon et al. (2019) who found that Caesalpinia legumes moved

854 frequently among succulent biomes on different continents, and only later shifted into

855 newly encountered biomes within each continent (Donoghue 2019). From our findings, we

856 suspect that ignoring paleobiome structure may cause the number of region-first transition

857 series to be underestimated. However, it must be borne in our minds that our results may

858 in part reflect the constraint built into our model that simultaneous shifts in biome and

859 region are not allowed (discussed below). In any case, explicitly testing for the effect of

860 paleobiome structure on event order will be important in evaluating patterns of supposed

861 phylogenetic biome conservatism (Crisp et al. 2009).

862

864 Although our model is simple, it is designed with certain statistical features that

865 would allow the model to be applied to diverse datasets beyond Viburnum, and to facilitate

866 extensions of the model towards more sophisticated designs. First, we treat many elements

867 in the evolutionary process as model parameters, whose values we estimate from the

868 phylogenetic dataset in question. For example, the $w$ parameters control which layers of

869 the paleobiome graphical structure are most relevant to the evolutionary process, and the

$870 y$ parameter controls how important weak structural features are when modeling dispersal

871 or biome shift events. Second, the Bayesian modeling framework we chose is ideal for

872 managing complex and parameter-rich hierarchical models (Höhna et al. 2016), allowing

873 for future models to explore the importance of other factors highlighted in the conceptual 
874 model of Donoghue and Edwards (2014) - geographical distance (Webb and Ree 2012),

875 region size (Tagliacollo et al. 2015), biome size and shared perimeter (Cardillo et al. 2017),

876 ecological distance (Meseguer et al. 2015), and the effect of biotic interactions on trait and

877 range evolution (Quintero and Landis 2019) — by introducing new parameterizations for

878 computing the time-stratified rate matrices, $Q(m)$. Our Bayesian framework is also capable

879 of handling sources of uncertainty in the paleobiome graphs, such as uncertainty in the

880 importance of various spatial features or in the age of the appearance of a biome within a

881 region (Landis et al. 2018).

882 In our application of the model to Viburnum, we defined only three biomes and six

883 regions, but the general framework translates to other biogeographical systems with

884 different regions and biomes, provided one can construct an adequate time series of

885 paleobiome graphs. Though our literature-based approach to paleobiome graph

886 construction was somewhat subjective, we found it to be the most integrative way to

887 summarize varied global biome reconstructions, as most individual studies are purely

888 qualitative (Wolfe 1985; Morley 2000; Jetz and Fine 2012; Willis and McElwain 2014;

889 Graham 2011; Graham 2018; but see Kaplan et al. 2003) and based on disparate lines of

890 paleoecological, paleoclimatological, and paleogeological evidence. We believe that our

891 paleobiome graphs for the Northern Hemisphere are sufficiently accurate to show that

892 spatial and temporal variation in the distribution of tropical, warm temperate, and cold

893 temperate forest biomes in space and time can influence how species ranges and biome

894 affinities evolve over time. Nonetheless, future studies should explore more quantitative

895 approaches to defining paleobiome structures for use with the time-stratified regional

896 biome shift model. 
Our simple model of regional biome shifts lacks several desired features. Perhaps

899 biome at a time when, in reality species may occupy multiple biomes and/or regions (e.g.,

900 Liquidambar styraciflua is a tree that thrives both in temperate deciduous forests of eastern

901 North America and in the cloud forests of Mexico). On paper, it is straightforward to extend

902 the concepts of this model to standard multi-character models, such as the Dispersal-

903 Extinction-Cladogenesis model (Ree et al. 2005; Matzke 2014; Sukumaran et al. 2015). As a

904 DEC model variant, lineages would be capable of gaining affinities with any biomes

905 available within their range. For example, for $M$ biomes and $N$ regions, there are on the

906 order of $2^{M+N}$ combinations of presences and absences across biomes and regions, and on

907 the order of $2^{M N}$ combinations if region-specific biome occupancies are considered.

908 Computationally, this creates a vast number of viable state combinations, many of which

909 cannot be eliminated from the state space (Webb and Ree 2012). Such a large state space

910 will hinder standard likelihood-based inference procedures for discrete biogeography (Ree

911 and Sanmartín 2009), though recent methodological advances addressing this problem

912 should prove useful (Landis et al. 2013; Quintero and Landis 2019).

913 Geographical state-dependent diversification (GeoSSE) models may also be

914 interfaced with our model. Incorporating the effect of biome availability on the extinction

915 rate would, at a minimum, be a very important contribution towards explaining patterns of

916 extant diversity. For example, tropical biomes have declined in dominance since the

917 Paleogene, and many ancient Viburnum lineages may have since gone extinct in the tropics,

918 perhaps owing to biotic interactions (the dying embers hypothesis of Spriggs et al. 2015).

919 In this sense, we expect that our model will overestimate how long a lineage may persist in 
920 a region that lacks the appropriate biome, since our model does not threaten ill-adapted

921 species with higher extinction rates. Efforts to extend GeoSSE models in this manner will

922 face similar, if not more severe, challenges to those encountered in the DEC framework,

923 both in terms of computational limits and numbers of parameters (Beaulieu and O'Meara

924 2016; Caetano et al. 2018).

925 If diversification rates vary conditionally on a lineage's biome-region state, then so

926 should the underlying divergence time estimates. At a minimum, one should jointly

927 estimate divergence times and diversification dynamics to correctly propagate uncertainty

928 in phylogenetic estimates through to ancestral state estimates (Höhna et al. 2019). Beyond

929 that, paleogeographically structured models of biogeography have been shown to be useful

930 for estimating divergence times (Landis 2017; Landis et al. 2018). Paleoecological models,

931 such as our Paleobiome model, could be useful in some cases, perhaps for dating clades

932 where some degree of phylogenetic niche conservatism can be safely assumed (Wiens and

933 Donoghue (2004); Crisp et al. (2009); but see Donoghue and Edwards (2014) for potential

934 pitfalls with this approach). For instance, Baldwin and Sanderson (1998) hypothesized that

935 continental tarweeds (Madiinae, Asteraceae) radiated within the seasonally dry California

936 Florisitic Province only after Miocene aridification created the province. Baldwin and

937 Sanderson then translated this relationship between biome age and biome affinity to date

938 the maximum crown age of tarweeds, and thus date the maximum crown age of a notable

939 radiation nested within the tarweeds, the Hawaiian silversword alliance. In the future,

940 rather than calibrating the age of tarweeds by asserting a paleoecological hypothesis, it

941 would be possible to use our biome shift model to measure the probability of the dry

942 radiation scenario against competing scenarios, thereby dating the tarweeds (or other 
943 clades) based on what ecological opportunities they made use of in different areas and at

944 different times (Baldwin and Sanderson 1998; Landis 2017; Landis et al. 2018).

945 Finally, although we have compared inferences of event series under several biome

946 structure models, and have argued that paleobiome models can influence such inferences,

947 we caution that event series themselves may not be accurate descriptors of some relevant

948 evolutionary scenarios. Notably, we reiterate that our model does not allow for

949 simultaneous shifts in both biome and biogeographic region. In other words, all biome

950 shifts are restricted to occur within a biogeographic region. This makes several important

951 assumptions about the processes involved in biome shifting. The first is that movements

952 between biomes are non-trivial for organisms, and require a period of time that allows a

953 founding population to adapt to its new environment. If this is true, we see two scenarios

954 that would likely describe the vast majority of biome shifts. First, if two biomes are

955 adjacent to one another, repeated propagule dispersal from the ancestral to the novel

956 biome could allow for gradual adaptation to a new habitat. Second, biome shifts could occur

957 "in-situ", as one major vegetation type gradually transforms into another, in place, as

958 climate change progresses (“lock-step” model of Edwards et al. 2017). Both of these

959 scenarios would occur within biogeographical regions, and we think they probably have

960 accounted for biome shifts in Viburnum, as well as many other lineages. However, it is also

961 possible that a shift into a new biome could occur during a transition from one region into

962 another. For example, a warm temperate-adapted lineage might adapt to colder forests

963 during range expansion through Beringia, or there might be long-distance dispersal of an

964 organism into newly encountered cold temperate forests to which it was pre-adapted.

965 Furthermore, it is important to note that our restriction of biome shifts to occur within 
966 biogeographical regions does not completely preclude long-distance dispersal biome shifts

967 within regions; nothing in our model ensures that ancestral and novel biomes are adjacent

968 to one another within a given region, especially given that the regions often used are very

969 large and environmentally heterogeneous. Such considerations highlight that the model we

970 have presented here is simplistic in some of its basic assumptions. We view it as a start in

971 the right direction, and look forward to extensions that will allow us to test a variety of

972 more nuanced hypotheses.

The potential for a lineage to adapt to new biomes depends in part on the

976 geographical opportunities that the lineage encountered in time and space. In the case of

977 Viburnum, we have shown that differing assumptions about the past distribution of biomes

978 can have a significant impact on ancestral biome estimates. And, when we integrate

979 information about the changing distribution of biomes through time, we favor an origin of

980 Viburnum in warm temperate or tropical forests, and confidently rule out an origin in cold

981 temperate forests. The confluence of this line of evidence with our analyses based instead

982 on fossil biome assignments (Landis et al. 2020) provides much greater confidence in a

983 result that orients our entire understanding of the direction of evolution and ecological

984 diversification in this clade.

985 More generally, we hope that our analyses will motivate biogeographers who wish

986 to estimate ancestral biomes to account for variation in the spatial distribution of biomes

987 through time. We also caution that phylogenetic estimates of ancestral biome affinities

988 derived entirely from extant taxa and biomes may be misleading, particularly for older 
989 lineages, even should standard statistical diagnostics indicate that the inference model fits

990 the data relatively well. While we have achieved some conceptual understanding of the

991 interplay between biome shifts in lineages and biome distributions over time, many

992 theoretical and statistical problems must still be solved for us to fully appreciate the

993 significance of changing biome availability in generating Earth's biodiversity. In presenting

994 our simple model, we hope to provoke further inquiry into how life diversified throughout

995 the biomes of an ever-changing planet.

FUNDING

999 and a Gaylord Donnelley Environmental Fellowship to MJL through the Yale Institute of

1000 Biospheric Studies. Our research on Viburnum has been funded through a series of NSF

1001 awards to MJD and EJE (most recently, DEB-1557059 and DEB-1753504), and in part

1002 through the Division of Botany of the Yale Peabody Museum of Natural History.

1005 We thank Bryan Carstens, Mark Holder, and two anonymous reviewers for their

1006 thoughtful comments. Ignacio Quintero, Nate Upham, David Polly, and Tracy Heath

1007 provided useful feedback that helped us frame the context of the research. We also wish to

1008 thank Paul Valdes, who provided us with various BIOME4 reconstructions. Finally, we

1009 express gratitude to the Donoghue, Edwards, and Near lab groups for supporting the

1010 development of this work since its inception. 
REFERENCES

1013 Baldwin, B. G. and M. J. Sanderson. 1998. Age and rate of diversification of the Hawaiian 1014 silversword alliance (Compositae). Proceedings of the National Academy of Sciences 1015 95: 9402-6.

1016 Baskin, J. M. and C. C. Baskin. 2016. Origins and Relationships of the Mixed Mesophytic 1017 Forest of Oregon-Idaho, China, and Kentucky: Review and Synthesis. Ann. Missouri $1018 \quad$ Bot. Gard. 101: 525-552.

1019 Beaulieu, J. M. and B. C. O’Meara. 2016. Detecting Hidden Diversification Shifts in Models of 1020 Trait-Dependent Speciation and Extinction. Systematic Biology 65: 583-601.

1021 Bielejec, F., P. Lemey, G. Baele, A. Rambaut, and M. A. Suchard. 2014. Inferring $1022 \quad$ Heterogeneous Evolutionary Processes Through Time: From Sequence Substitution 1023 to Phylogeography. Systematic Biology 63: 493-504.

1024 Buerki, S., F. Forest, N. Alvarez, J. A. A. Nylander, N. Arrigo, and I. Sanmartín. 2011. An

1025 Evaluation of New Parsimony-Based Versus Parametric Inference Methods in 1026 Biogeography: A Case Study Using the Globally Distributed Plant Family 1027 Sapindaceae. Journal of Biogeography 38: 531-50.

1028 Caetano, D. S., B. C. O’Meara, and J. M. Beaulieu. 2018. Hidden State Models Improve State1029 Dependent Diversification Approaches, Including Biogeographical Models. Evolution $1030 \quad 72: 2308-24$.

1031 Cardillo, M., P. H. Weston, Z. K. M. Reynolds, P. M. Olde, A. R. Mast, E. M. Lemmon, A. R. 1032 Lemmon, and L. Bromham. 2017. The phylogeny and biogeography of Hakea 1033 (Proteaceae) reveals the role of biome shifts in a continental plant radiation. $1034 \quad$ Evolution 71: 1928-43. 
1035 Chatelet, D. S., W. L. Clement, L. Sack, M. J. Donoghue, and E. J. Edwards. 2013. The Evolution of Photosynthetic Anatomy in Viburnum (Adoxaceae). International Journal of Plant

$1037 \quad$ Sciences 174: 1277-91.

1038 Clement, W. L., M. Arakaki, P. W. Sweeney, E. J. Edwards, and M. J. Donoghue. 2014. A

1039 Chloroplast Tree for Viburnum (Adoxaceae) and Its Implications for Phylogenetic

1040 Classification and Character Evolution. American Journal of Botany 101: 1029-49.

1041 Crisp, M. D., M. T. K. Arroyo, L. G. Cook, M. A. Gandolfo, G. J. Jordan, M. S. McGlone, P. H.

1042 Weston, M. Westoby, P. Wilf, and P. H. Linder. 2009. Phylogenetic Biome

1043 Conservatism on a Global Scale. Nature 458: 754-56.

1044 Donoghue, M. J. 2008. A Phylogenetic Perspective on the Distribution of Plant Diversity.

$1045 \quad$ Proceedings of the National Academy of Sciences 105: 11549-55.

1046 Donoghue, M. J. 2019. Adaptation Meets Dispersal: Legumes in the Land of Succulents. New

$1047 \quad$ Phytologist 222: 1667-9.

1048 Donoghue, M. J., and E. J. Edwards. 2014. Biome Shifts and Niche Evolution in Plants. Annual

$1049 \quad$ Review of Ecology, Evolution, and Systematics 45: 547-72.

1050 Donoghue, M. J. and M. J. Sanderson. 2015. Confluence, Synnovation, and Depauperons in 1051 Plant Diversification. New Phytologist 207: 260-74.

1052 Eaton, D. A. R., E. L. Spriggs, B. Park, and M. J. Donoghue. 2017. Misconceptions on Missing 1053 Data in RAD-Seq Phylogenetics with a Deep-Scale Example from Flowering Plants. $1054 \quad$ Systematic Biology 66: 399-412.

1055 Edwards, E. J., D. S. Chatelet, B. Chen, J. Y. Ong, S. Tagane, H. Kanemitsu, K. Tagawa, K. 1056 Teramoto, B. Park, K.-F. Chung, J.-M. Hu, T. Yahara, and M. J. Donoghue. 2017. 

American Naturalist 190: S87-S104.

1059 Edwards, E. J., J. M. de Vos, and M. J. Donoghue. 2015. Doubtful Pathways to Cold Tolerance in Plants. Nature 521: E5.

1061 Felsenstein, J. 1981. Evolutionary Trees from DNA Sequences: A Maximum Likelihood Approach. Journal of Molecular Evolution 17: 368-76.

1063 Fine, P. V. A. and R. H. Ree. 2006. Evidence for a Time-Integrated Species-Area Effect on the Latitudinal Gradient in Tree Diversity. The American Naturalist 168: 796-804.

1065 Gagnon, E., J. J. Ringelberg, A. Bruneau, G. P. Lewis, and C. E. Hughes. 2019. Global Succulent Biome Phylogenetic Conservatism Across the Pantropical Caesalpinia Group

1067 (Leguminosae). New Phytologist 222: 1994-2008.

1068 Godfrey, C., M. Fan, G. Jesmok, D. Upadhyay, and A. Tripati. 2018. Petrography and stable

1069 isotope geochemistry of Oligocene-Miocene continental carbonates in south Texas:

1070 Implications for paleoclimate and paleoenvironment near sea-level. Sedimentary

$1071 \quad$ Geology 367: 69-83.

1072 Goldberg, E. E., K. Roy, R. Lande, and D. Jablonski. 2005. Diversity, endemism, and age

1073 distributions in macroevolutionary sources and sinks. The American Naturalist 165:

$1074 \quad 623-33$.

1075 Graham, A. 2011. A Natural History of the New World: The Ecology and Evolution of Plants in 1076 the Americas. University of Chicago Press.

1077 Graham, A. 2018. Land Bridges Ancient Environments, Plant Migrations, and New World 1078 Connections. University of Chicago Press. 
1079 Heath, T. A., J. P. Huelsenbeck, and T. Stadler. 2014. The Fossilized Birth-Death Process for

1080 Coherent Calibration of Divergence-Time Estimates. Proceedings of the National

$1081 \quad$ Academy of Sciences 111: E2957-E2966.

1082 Herold, N., J. Buzan, M. Seton, A. Goldner, J. A. M. Green, R. D. Müller, P. Markwick, and M.

1083 Huber. 2014. A suite of early Eocene ( $55 \mathrm{Ma})$ climate model boundary conditions.

1084 Geoscientific Model Development 7: 2077-90.

1085 Höhna, S., W. A. Freyman, Z. Nolen, J. P. Huelsenbeck, M. R. May, and B. R. Moore. 2019. A

1086 Bayesian Approach for Estimating Branch-Specific Speciation and Extinction Rates.

1087 bioRxiv. https://doi.org/10.1101/555805.

1088 Höhna, S., M. J. Landis, T. A. Heath, B. Boussau, N. Lartillot, B. R. Moore, J. P. Huelsenbeck,

1089 and F. Ronquist. 2016. RevBayes: Bayesian Phylogenetic Inference Using Graphical

1090 Models and Interactive Model-Specification Language. Systematic Biology 65: 726-

109136.

1092 Höhna, S., M. J. Landis, and J. P. Huelsenbeck. 2017. Parallel power posterior analyses for

1093 fast computation of marginal likelihoods in phylogenetics. BioRxiv 104422.

1094 Huelsenbeck, J. P., R. Nielsen, and J. P. Bollback. 2003. Stochastic mapping of morphological

$1095 \quad$ characters. Syst. Biol. 52: 131-158.

1096 Jeffreys, H. 1935. Some tests of significance, treated by the theory of probability. Proc.

1097 Camb. Phil. Soc. 31: 203-222.

1098 Jeffreys, H. 1961. Theory of Probability. Oxford: Oxford University Press.

1099 Jetz, W. and P. V. A. Fine. 2012. Global Gradients in Vertebrate Diversity Predicted by

$1100 \quad$ Historical Area-Productivity Dynamics and Contemporary Environment. PLoS

$1101 \quad$ Biology 10: e1001292. 
1102 Kaplan, J. O., N. H. Bigelow, I. C. Prentice, S. P. Harrison, P. J. Bartlein, T. R. Christensen, W.

1103 Cramer, et al. 2003. Climate change and Arctic ecosystems: 2. Modeling, paleodata-

1104 model comparisons, and future projections. Journal of Geophysical Research:

$1105 \quad$ Atmospheres 108 (D19).

1106 Klaus, K. V. and N. J. Matzke. 2019. Statistical Comparison of Trait-Dependent

1107 Biogeographical Models Indicates That Podocarpaceae Dispersal Is Influenced by

1108 Both Seed Cone Traits and Geographical Distance. Systematic Biology 69: 61-75.

1109 Kosiol, C., I. Holmes, and N. Goldman. 2007. An empirical codon model for protein sequence

$1110 \quad$ evolution. Mol. Biol. Evol. 24: 1464-1479.

1111 Landis, M. J. 2017. Biogeographic Dating of Speciation Times Using Paleogeographically

1112 Informed Processes. Systematic Biology 66: 128-44.

1113 Landis, M. J., D. A. R. Eaton, W. L. Clement, B. Park, E. L. Spriggs, P. W. Sweeney, E. J.

1114 Edwards, and M. J. Donoghue. 2020. Joint Phylogenetic Estimation of Geographic

1115 Movements and Biome Shifts During the Global Diversification of Viburnum.

1116 Systematic Biology. Advance online publication. doi.org/10.1093/sysbio/syaa027

1117 Landis, M. J., W. A. Freyman, and B. G. Baldwin. 2018. Retracing the Hawaiian silversword

1118 radiation despite phylogenetic, biogeographic, and paleogeographic uncertainty.

$1119 \quad$ Evolution 72: 2343-59.

1120 Landis, M. J., N. J. Matzke, B. R. Moore, and J. P. Huelsenbeck. 2013. Bayesian Analysis of

1121 Biogeography When the Number of Areas Is Large. Systematic Biology 62: 789-804.

1122 Latham, R. E. and R. E. Ricklefs. 1993. Continental Comparisons of Temperate-Zone Tree

1123 Species Diversity. Species Diversity in Ecological Communities: Historical and

1124 Geographical Perspectives, 294-314. 
1125 Lens, F., R. A. Vos, G. Charrier, T. van der Niet, V. Merckx, P. Baas, J. Aguirre Gutierrez, et al. 2016. Scalariform-to-Simple Transition in Vessel Perforation Plates Triggered by Differences in Climate During the Evolution of Adoxaceae. Annals of Botany 118:

Lu, L., P. W. Fritsch, N. J. Matzke, H. Wang, K. A. Kron, D. Z. Li, and J. J. Wiens. 2019. Why Is Fruit Colour so Variable? Phylogenetic Analyses Reveal Relationships Between Fruit-Colour Evolution, Biogeography and Diversification. Global Ecology and

1133 Matos-Maraví, P., N. J. Matzke, F. J. Larabee, R. M. Clouse, W. C. Wheeler, D. M. Sorger, A. V. Suarez, and M. Janda. 2018. Taxon Cycle Predictions Supported by Model-Based Inference in Indo-Pacific Trap-Jaw Ants (Hymenoptera: Formicidae: Odontomachus).

1137 Matzke, N. J. 2014. Model Selection in Historical Biogeography Reveals That Founder-Event Speciation Is a Crucial Process in Island Clades. Systematic Biology 63: 951-70.

1139 Meseguer, A. S., J. M. Lobo, R. Ree, D. J. Beerling, and I. Sanmartín. 2015. Integrating Fossils, Phylogenies, and Niche Models into Biogeography to Reveal Ancient Evolutionary

1142 Morley, R. J. 2000. Origin and Evolution of Tropical Rain Forests. John Wiley \& Sons.

1143 Moss, J. and M. Tveten. 2019. kdensity: An R package for kernel density estimation with parametric starts and asymmetric kernels. Journal of Open Source Software 4: 1566.

1145 Mucina, L. 2019. Biome: Evolution of a Crucial Ecological and Biogeographical Concept. New $1146 \quad$ Phytologist 222: 97-114. 
1147 Olson, D. M., E. Dinerstein, E. D. Wikramanayake, N. D. Burgess, G. V. N. Powell, E. C.

1148 Underwood, J. A. D’amico, et al. 2001. Terrestrial Ecoregions of the World: A New Map of Life on Earth a New Global Map of Terrestrial Ecoregions Provides an

Pagel, M. 1994. Detecting Correlated Evolution on Phylogenies: A General Method for the Innovative Tool for Conserving Biodiversity. BioScience 51: 933-38.

1154 Pound, M. J., A. M. Haywood, U. Salzmann, and J. B. Riding. 2012. Global Vegetation Dynamics and Latitudinal Temperature Gradients During the Mid to Late Miocene

1157 Pound, M. J., A. M. Haywood, U. Salzmann, J. B. Riding, D. J. Lunt, and S. J. Hunter. 2011. A Tortonian (Late Miocene, 11.61 - 7.25 Ma) Global Vegetation Reconstruction.

1160 Pound, M. J., and U. Salzmann. 2017. Heterogeneity in Global Vegetation and Terrestrial Palaeogeography, Palaeoclimatology, Palaeoecology 300: 29-45.

1163 Prentice, I. C., W. Cramer, S. P. Harrison, R. Leemans, R. A. Monserud, and A. M. Solomon. 1992. A Global Biome Model Based on Plant Physiology and Dominance, Soil 1165 Properties and Climate. Journal of Biogeography, 117-34.

1166 Quintero, I. and M. J. Landis. 2019. Interdependent Phenotypic and Biogeographic Evolution 1167 Driven by Biotic Interactions. Systematic Biology. Advance online publication. doi.org/10.1093/sysbio/syz082 
1169 Ree, R. H., B. R. Moore, C. O. Webb, and M. J. Donoghue. 2005. A Likelihood Framework for Inferring the Evolution of Geographic Range on Phylogenetic Trees. Evolution 59: 2299-2311.

1172 Ree, R. H. and I. Sanmartín. 2009. Prospects and Challenges for Parametric Models in Historical Biogeographical Inference. Journal of Biogeography 36: 1211-20.

1174 Ree, R. H. and S. A. Smith. 2008. Maximum Likelihood Inference of Geographic Range Evolution by Dispersal, Local Extinction, and Cladogenesis. Systematic Biology 57: 4-

1177 Rodrigue, N., H. Philippe, and N. Lartillot. 2008. Uniformization for sampling realizations of Markov processes: applications to Bayesian implementations of codon substitution models. Bioinformatics. 24: 56-62.

1180 Ronquist, F., S. Klopfstein, L. Vilhelmsen, S. Schulmeister, D. L. Murray, and A. P. Rasnitsyn. 2012. A total-evidence approach to dating with fossils, applied to the early radiation

1183 Salzmann, U., A. M. Haywood, and D. J. Lunt. 2009. The past is a guide to the future?

1184 Comparing Middle Pliocene vegetation with predicted biome distributions for the 1185 twenty-first century. Philosophical Transactions: Series A: Mathematical, Physical, $1186 \quad$ and Engineering Sciences 367: 189-204.

1187 Salzmann, U., A. M. Haywood, D. J. Lunt, P. J. Valdes, and D. J. Hill. 2008. A new global biome reconstruction and data-model comparison for the Middle Pliocene. Global Ecology and Biogeography 17: 432-47.

1190 Schmerler, S. B., W. L. Clement, J. M. Beaulieu, D. S. Chatelet, L. Sack, M. J. Donoghue, and E. J. 1191 Edwards. 2012. Evolution of Leaf Form Correlates with Tropical-Temperate 
Transitions in Viburnum (Adoxaceae). Proceedings of the Royal Society B: Biological Sciences 279: 3905-13.

1194 Scoffoni, C., D. S. Chatelet, J. Pasquet-Kok, M. Rawls, M. J. Donoghue, E. J. Edwards, and L. Sack. 2016. Hydraulic Basis for the Evolution of Photosynthetic Productivity. Nature Plants 2: 16072.

1197 Spriggs, E. L., W. L. Clement, P. W. Sweeney, S. Madriñán, E. J. Edwards, and M. J. Donoghue. 1198 2015. Temperate Radiations and Dying Embers of a Tropical Past: The Diversification of Viburnum. New Phytologist 207: 340-54.

1200 Sukumaran, J., E. P. Economo, and L. L. Knowles. 2015. Machine Learning Biogeographic $1201 \quad$ Processes from Biotic Patterns: A New Trait-Dependent Dispersal and Diversification Model with Model Choice by Simulation-Trained Discriminant 1203 Analysis. Systematic Biology 65: 525-545.

1204 Sukumaran, J. and L. L. Knowles. 2018. Trait-Dependent Biogeography:(Re) Integrating Biology into Probabilistic Historical Biogeographical Models. Trends in Ecology \& $1206 \quad$ Evolution 33: 390-98.

1207 Tagliacollo, V. A., S. M. Duke-Sylvester, W. A. Matamoros, P. Chakrabarty, and J. S. Albert. 1208 2015. Coordinated Dispersal and Pre-Isthmian Assembly of the Central American 1209 Ichthyofauna. Systematic Biology 66: 183-96.

1210 Tiffney, B. H. 1985a. Perspectives on the origin of the floristic similarity between eastern 1211 Asia and eastern North America. J. Arnold Arbor. 66: 73-94.

1212 Tiffney, B. H. 1985b. The Eocene North Atlantic Land Bridge: Its importance in Tertiary and 1213 modern phytogeography of the Northern Hemisphere. J. Arnold Arbor. 66: 243-273. 
1214 Tiffney, B. H. and S. R. Manchester. 2001. The use of geological and paleontological evidence

1215

1216

1217

1218

1219

1220

1221

1222

1223

1224

1225

1226

1227

1228

1229

1230

1231

1232

1233

1234

1235

1236 in evaluating plant phylogeographic hypotheses in the Northern Hemisphere Tertiary. Int. J. Plant. Sci. 162: S3-S17.

Verdinelli, I. and L. Wasserman. 1995. Computing Bayes Factors Using a Generalization of the Savage-Dickey Density Ratio. Journal of the American Statistical Association 90: 614-18.

Webb, C. O. and R. H. Ree. 2012. Historical Biogeography Inference in Malesia. In Biotic Evolution and Environmental Change in Southeast Asia, edited by D. Gower, K. Johnson, J. Richardson, B. Rosen, L. Ruber, and S. Williams, 191-215. Cambridge University Press.

Weeks, A., F. Zapata, S. K. Pell, D. C. Daly, J. D. Mitchell, and P. V. A. Fine. 2014. To Move or to Evolve: Contrasting Patterns of Intercontinental Connectivity and Climatic Niche Evolution in 'Terebinthaceae' (Anacardiaceae and Burseraceae). Frontiers in Genetics 5: 409.

Whittaker, R. H. 1970. Communities and Ecosystems. Macmillan Company.

Wickham, H. 2016. ggplot2: Elegant Graphics for Data Analysis. Springer.

Wiens, J. J. and M. J. Donoghue. 2004. Historical Biogeography, Ecology and Species Richness. Trends in Ecology \& Evolution 19: 639-44.

Willis, K. and J. McElwain. 2014. The Evolution of Plants. Oxford University Press.

Wolfe, J. A. 1975. Some aspects of the plant geography of the Northern Hemisphere during the Late Cretaceous and Tertiary. Ann. Missouri Bot. Gard. 62: 264-279.

Wolfe, J. A. 1985. Distribution of Major Vegetational Types During the Tertiary. The Carbon Cycle and Atmospheric CO2: Natural Variations Archean to Present 32: 357-75. 
bioRxiv preprint doi: https://doi.org/10.1101/832527; this version posted June 1,2020. The copyright holder for this preprint (which was not certified by peer review) is the author/funder, who has granted bioRxiv a license to display the preprint in perpetuity. It is made available under aCC-BY 4.0 International license.

1237 Xie, W., P. O. Lewis, Y. Fan, L. Kuo, and M.-H. Chen. 2011. Improving marginal likelihood estimation for Bayesian phylogenetic model selection. Syst. Biol. 60: 150-160. 
1241 Table 1. Regional biome shift parameter estimates. Posterior median estimates are in bold

1242 and 95\% highest posterior densities are in brackets. Fixed parameters under the Null

1243 Biome analysis do not have brackets.

\section{FIGURE CAPTIONS}

1247 Figure 1. Cartoon of the relationship between paleobiome structure and a regional biome

1248 shift process. The left and right panels are aligned to the same geological time scale that is

1249 divided into a Hot (red) interval followed by a Cold (blue) interval. (A) Maps of paleobiome

1250 structure with two regions, East (E) and West (W), and two focal biomes of interest, Hot

1251 (H) and Cold (C), in which the expansive Hot biome is replaced by the Cold biome as the

1252 East and West regions separate. (B) Paleobiome adjacency matrices encode the availability

1253 of biomes within regions and the connectivity of biomes between regions based on

1254 whether paleobiome features are strong (dark) or weak (light). Diagonal elements reflect

1255 biome availability within regions while off-diagonal elements report biome connectivity

1256 between regions. (C) Two possible regional biome shift histories for a phylogeny with a

1257 western, hot-adapted (HW) origin. Lineages shift between biomes at rates that depend on

1258 the availability of biomes within the lineage's current region and disperse between regions

1259 at rates that depend on connectivity of the lineage's current biome between regions. The

1260 two histories require (top) or do not require (bottom) evolutionary events to be congruent 
1261 with paleobiome structure. (D) Time-dependent biome shift rates for the four possible

1262 events: HW to CW, CW to HW, HE to CE, and CE to HE. (E) Time-dependent dispersal rates

1263 for the four possible events: HW to HE, HE to HW, CW to CE, and CE to CW.

1264

1265 Figure 2. Availability and connectivity of biomes from Late Cretaceous (100 Ma) to Recent.

1266 Adjacency matrices are used to structure the time-stratified phylogenetic biome shift

1267 process. Rows correspond to eight major time intervals, while columns correspond to

1268 regional features, specifically uninformative features (black), simple geographical features

1269 (brown), or features involving the tropical (red), warm temperate (green), and cold

1270 temperate (blue) forest biomes. The matrix for each time and feature encodes the

1271 availability of (the diagonal) and the connectivity between (off-diagonal) regions for that

1272 feature at that time, where matrix rows and columns correspond to source and destination

1273 regions, respectively. Cells representing availability and connectivity are shaded to

1274 represent those features as being strong (dark), weak (medium), or marginal (light).

1275

1276 Figure 3. Stationary distribution of biome-region states under the paleobiome model. The

1277 stationary probabilities across biome-regions (y-axis) vary with respect to time ( $\mathrm{x}$-axis).

1278 Stationary probabilities were computed assuming that biome and region shifts are

1279 expected to occur in equal proportion $(\beta=\delta=0.5)$, that lineages tend to shift and

1280 disperse in a manner than depends heavily on the paleobiome structure $\left(w_{U}=0.04, w_{G}=\right.$

12810.16 , and $w_{B}=0.8$ ), and that biomes with strong presences primarily define the structure

1282 of biome graphs $(y=0.1)$. Parameters were chosen to show interesting variation. Note, all

1283 stationary probabilities would be equal over all times if $w_{U}=1$. 
1285 Figure 4. Simulation experiment results. One hundred datasets were simulated under five

1286 conditions that varied the strength of $w_{B}$, then fitted to the paleobiome model to assess

1287 model performance. (A) Markers show the true simulated strength for $w_{B}$ (closed square),

1288 the posterior median values estimated from simulated replicates (open circles), the median

1289 of those posterior medians (closed circle), and the upper and lower bounds of the 95\%

1290 highest posterior density (open triangles). The coverage frequency reports the proportion

1291 of simulation analyses in which the simulating value of $w_{B}$ is falls within the $95 \%$ highest

1292 posterior density. (B) Bars report the proportions of simulated datasets that supported the

1293 model where $w_{B}>0$, categorized by the strength of that support in terms of Bayes factors

1294 (Jeffreys 1961).

1295

1296 Figure 5. Ancestral biome-region state estimates for Viburnum. Estimates produced under

1297 (A) Paleobiome, (B) Modern Biome, and (C) Null Biome settings. Colored pie charts report

1298 posterior support for the three most probable biome-region states per node. Pie charts for

1299 root state probabilities are magnified to improve visibility. Vertical white and gray bands

1300 correspond to major geological timeframes referenced in this study.

1302 Figure 6. Ancestral proportions of lineage state frequencies through time for Viburnum. The

1303 left column (A-C) shows the lineages biome-region states, where regions differ by color

1304 and biomes differ by shading (see legend). Proportions of reconstructed lineages in each

1305 biome-region state are shown for estimates under the Paleobiome (A), Modern Biome (B),

1306 and Null Biome (C) settings. The right column (D-F) shows the proportion of lineages with 
1307 biome states that match (dark) or mismatch (light) the non-marginal biomes that are

1308 locally accessible given any lineage's location, as defined under the Paleobiome structure

1309 (see main text for details). Proportions of reconstructed lineages with biome match and

1310 mismatch scores are shown for estimates under the Paleobiome (D), Modern Biome (E), and

1311 Null Biome (F) settings.

1312

1313 Figure 7. Stationary probabilities for the Viburnum root state during the Late Cretaceous.

1314 Posterior stationary probabilities for $\pi\left(m_{\text {root }}\right)$ are given for each biome structure model

1315 (grouped rows) and for each biome-region state (colors) as posterior means (points) and

1316 credible intervals (HPD80, thick lines; HPD95, thin lines).

1318 Figure 8. Proportions of inferred events and event series across major time intervals for

1319 Viburnum. Posterior proportions are presented as posterior means (points) and credible

1320 intervals (HPD80, thick lines; HPD95, thin lines). The left column (A-C) presents the

1321 proportions of estimated biome shift and dispersal events with respect to time, showing

1322 only the eight biome shift and dispersal events among the warm and cold temperate forests

1323 of East Asia and North America. Proportions of events are shown for inferences under the

1324 Paleobiome (A), Modern Biome (B), and Null Biome (C) settings. The right column (D-F)

1325 shows the proportions of the six types of event series with respect to time (defined in main

1326 text). Each event series type is labeled with a 'state triplet' to indicate either transitions in

1327 the biome $(\mathrm{A}, \mathrm{B}, \mathrm{C})$ or region $(\mathrm{X}, \mathrm{Y}, \mathrm{Z})$ state. 
1330 Table 1

Biome structure

\begin{tabular}{|c|c|c|c|}
\hline Parameter & Paleo & Modern & Null \\
\hline \multirow[t]{2}{*}{$\mu$} & 0.06 & 0.05 & 0.03 \\
\hline & {$[0.03,0.10]$} & {$[0.03,0.09]$} & {$[0.02,0.06]$} \\
\hline \multirow[t]{2}{*}{$f_{\beta}$} & 0.85 & 0.83 & 0.92 \\
\hline & {$[0.75,0.94]$} & {$[0.69,0.93]$} & {$[0.85,0.97]$} \\
\hline \multirow[t]{2}{*}{$f_{\delta}$} & 0.15 & 0.17 & 0.08 \\
\hline & {$[0.06,0.25]$} & {$[0.07,0.31]$} & {$[0.03,0.15]$} \\
\hline \multirow{2}{*}{$\beta_{T W}$} & 0.67 & 0.50 & 0.54 \\
\hline & {$[0.20,1.00]$} & {$[0.05,0.95]$} & {$[0.10,1.00]$} \\
\hline \multirow{2}{*}{$\beta_{W C}$} & 0.81 & 0.81 & 0.74 \\
\hline & {$[0.47,1.00]$} & {$[0.49,1.00]$} & {$[0.39,1.00]$} \\
\hline \multirow{2}{*}{$\beta_{W T}$} & 0.29 & 0.39 & 0.31 \\
\hline & {$[0.10,0.62]$} & {$[0.11,0.85]$} & {$[0.06,0.64]$} \\
\hline \multirow{2}{*}{$\beta_{C W}$} & 0.38 & 0.65 & 0.71 \\
\hline & {$[0.01,0.80]$} & {$[0.34,1.00]$} & {$[0.33,1.00]$} \\
\hline \multirow{2}{*}{$w_{U}$} & 0.01 & 0.02 & \multirow{2}{*}{1} \\
\hline & {$[0.00,0.07]$} & {$[0.00,0.08]$} & \\
\hline \multirow{2}{*}{$w_{G}$} & 0.04 & 0.04 & \multirow{2}{*}{$\mathbf{0}$} \\
\hline & {$[0.00,0.18]$} & {$[0.00,0.20]$} & \\
\hline \multirow{2}{*}{$w_{B}$} & 0.94 & 0.93 & \multirow{2}{*}{$\mathbf{0}$} \\
\hline & {$[0.78,1.00]$} & {$[0.76,1.00]$} & \\
\hline \multirow{2}{*}{$\mathrm{y}$} & 0.65 & 0.52 & \multirow{2}{*}{1} \\
\hline & {$[0.27,0.99]$} & {$[0.09,0.95]$} & \\
\hline
\end{tabular}




\section{Figure 1}
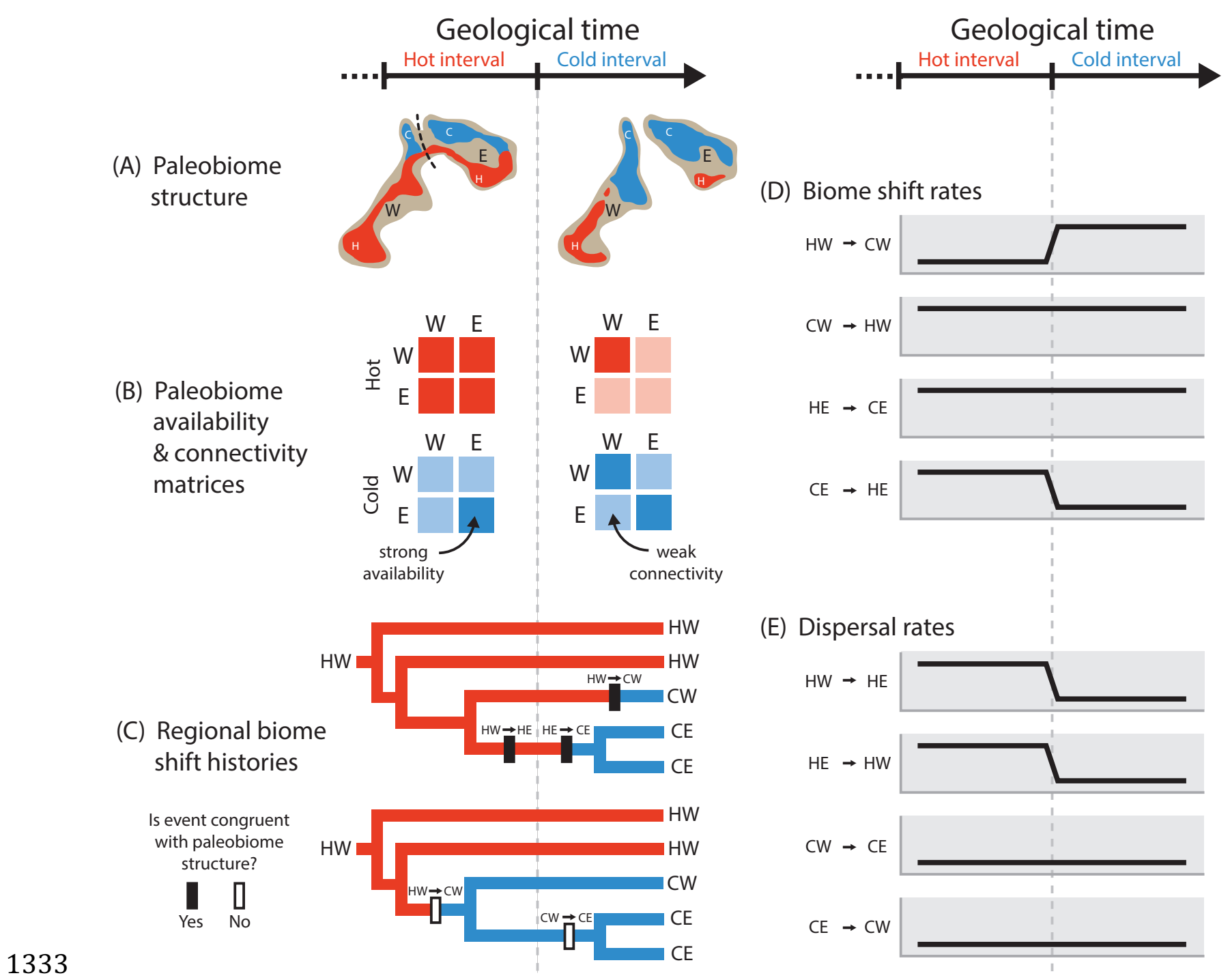
bioRxiv preprint doi: https://doi.org/10.1101/832527; this version posted June 1, 2020. The copyright holder for this preprint (which was not certified by peer review) is the author/funder, who has granted bioRxiv a license to display the preprint in perpetuity. It is made available under aCC-BY 4.0 International license.

\section{Figure 2}
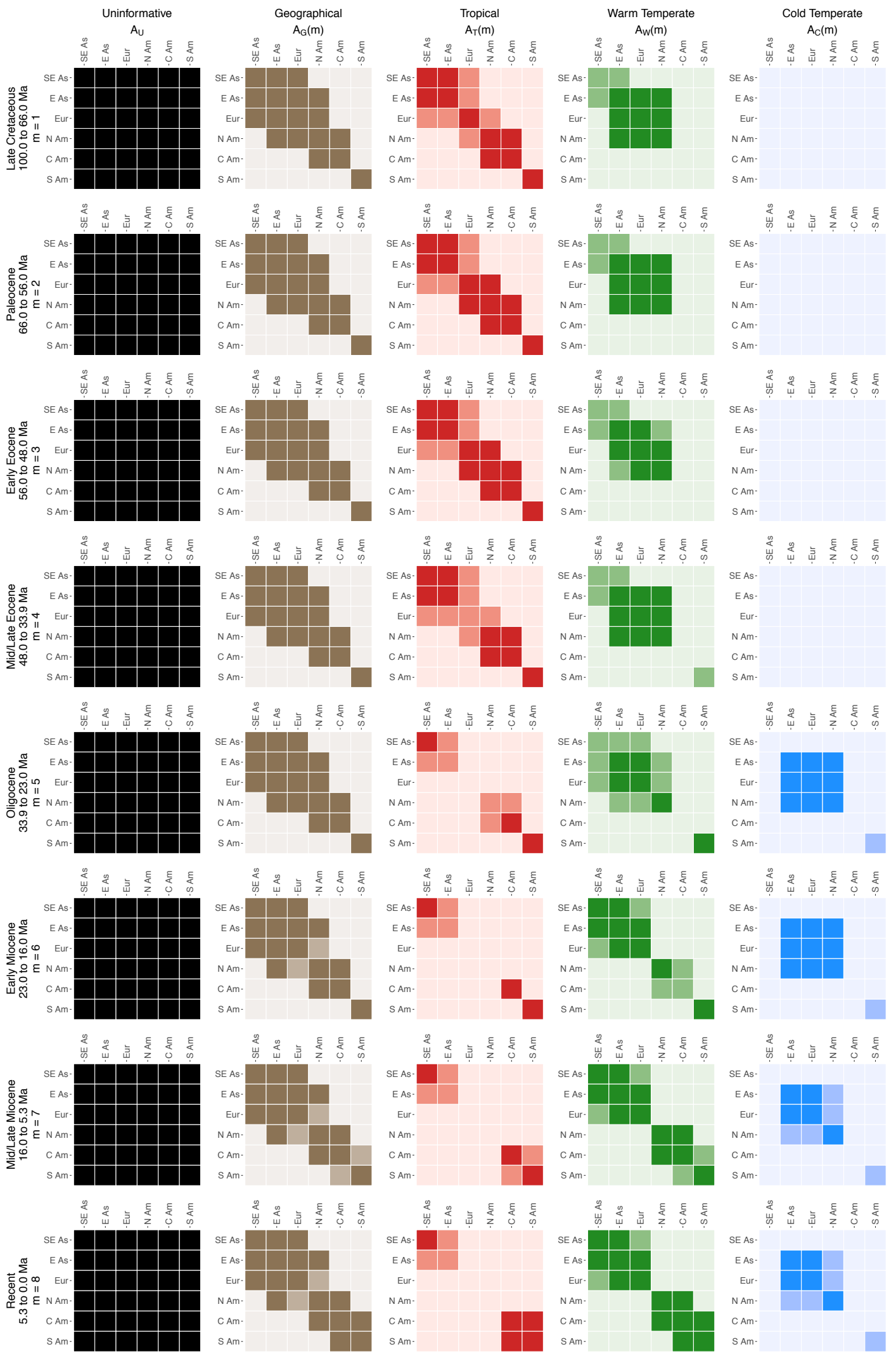
bioRxiv preprint doi: https://doi.org/10.1101/832527; this version posted June 1, 2020. The copyright holder for this preprint (which was not certified by peer review) is the author/funder, who has granted bioRxiv a license to display the preprint in perpetuity. It is made available under aCC-BY 4.0 International license.

\section{Figure 3}

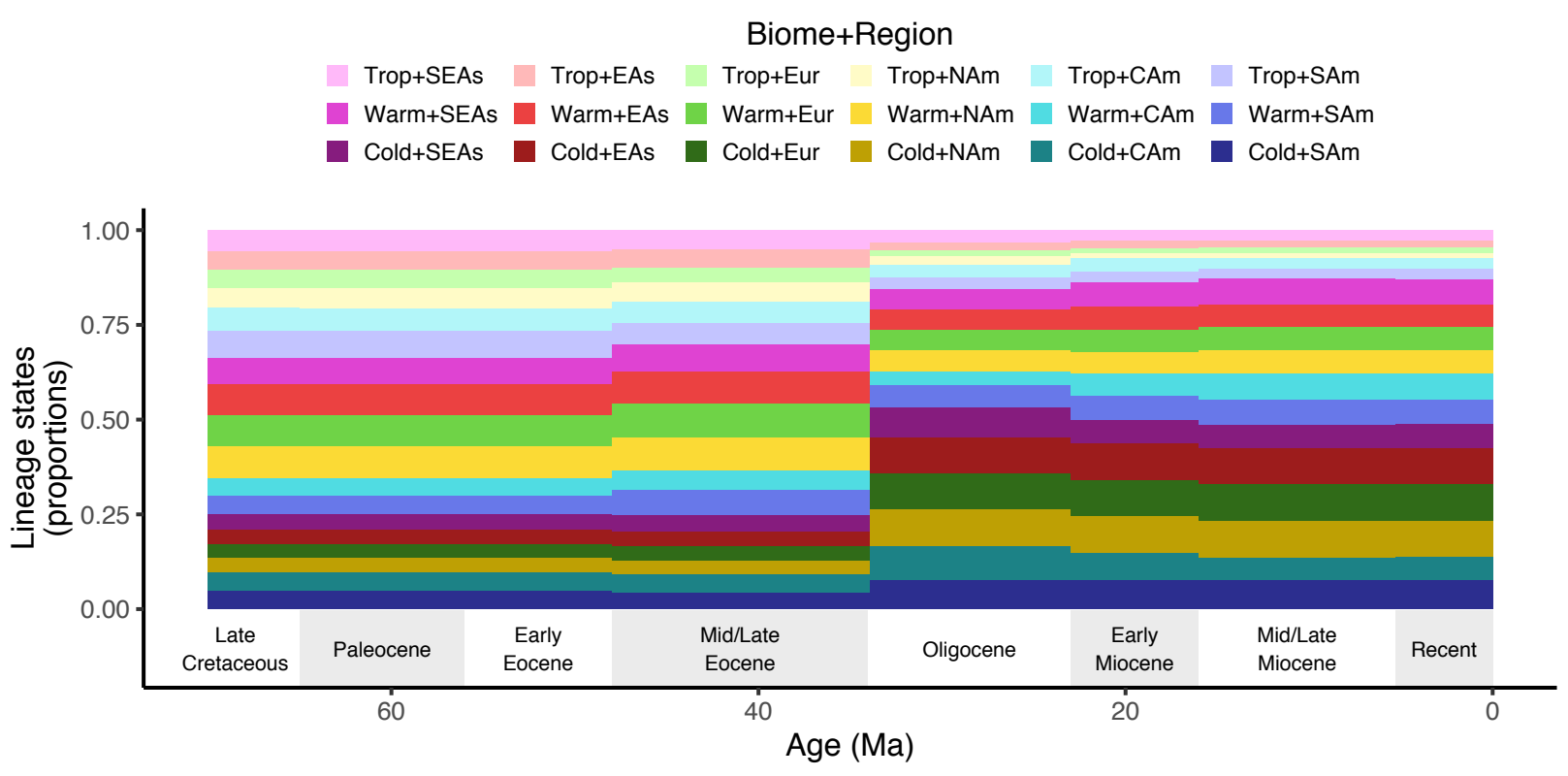


bioRxiv preprint doi: https://doi.org/10.1101/832527; this version posted June 1, 2020. The copyright holder for this preprint (which was not certified by peer review) is the author/funder, who has granted bioRxiv a license to display the preprint in perpetuity. It is made available under aCC-BY 4.0 International license.

\section{Figure 4}
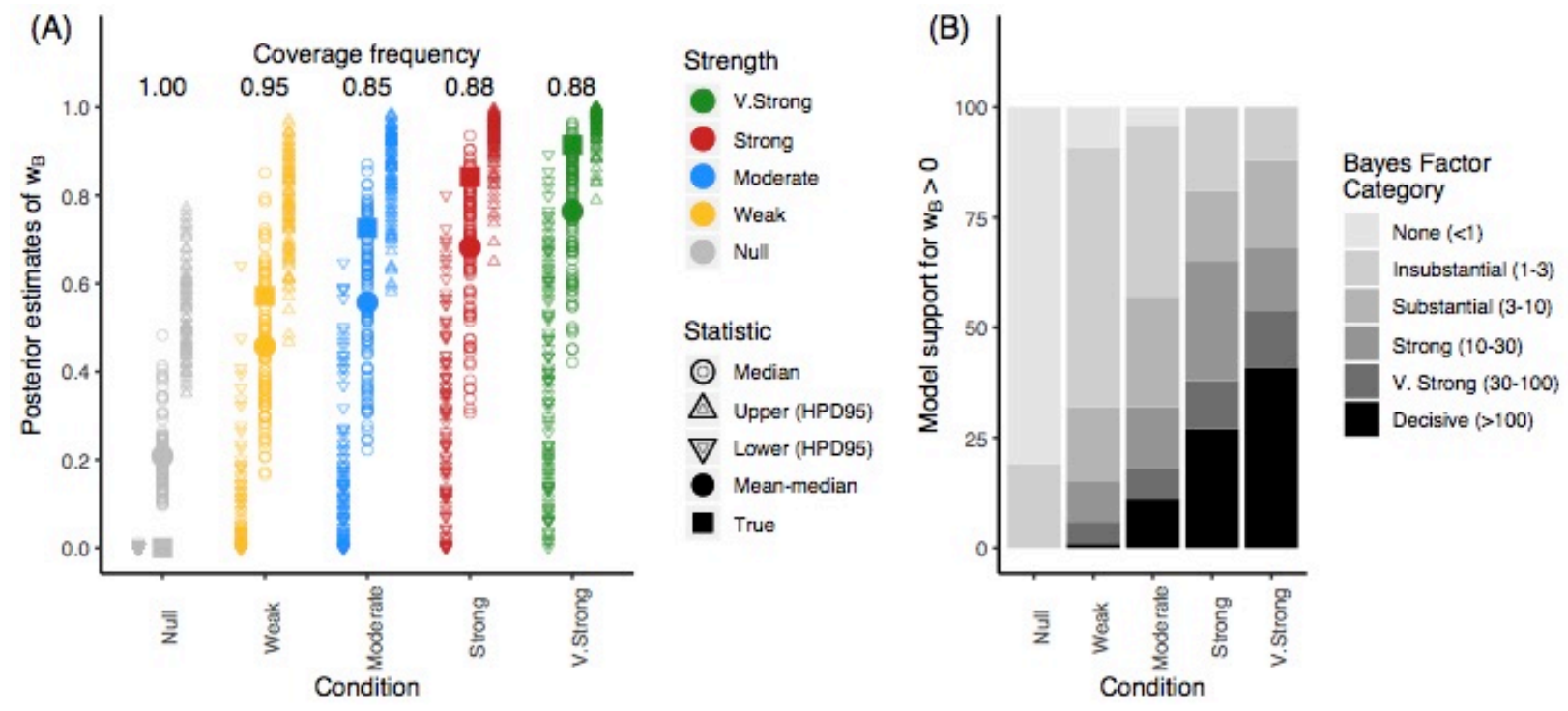
bioRxiv preprint doi: https://doi org/10.1101/832527; this version posted June 1,2020. The copyright holder for this preprint (which was not certified by peer review) is the author/funder, who has granted bioRxiv a license to display the preprint in perpetuity. It is made available under aCC-BY 4.0 International license.

\section{$1340 \quad$ Figure 5}
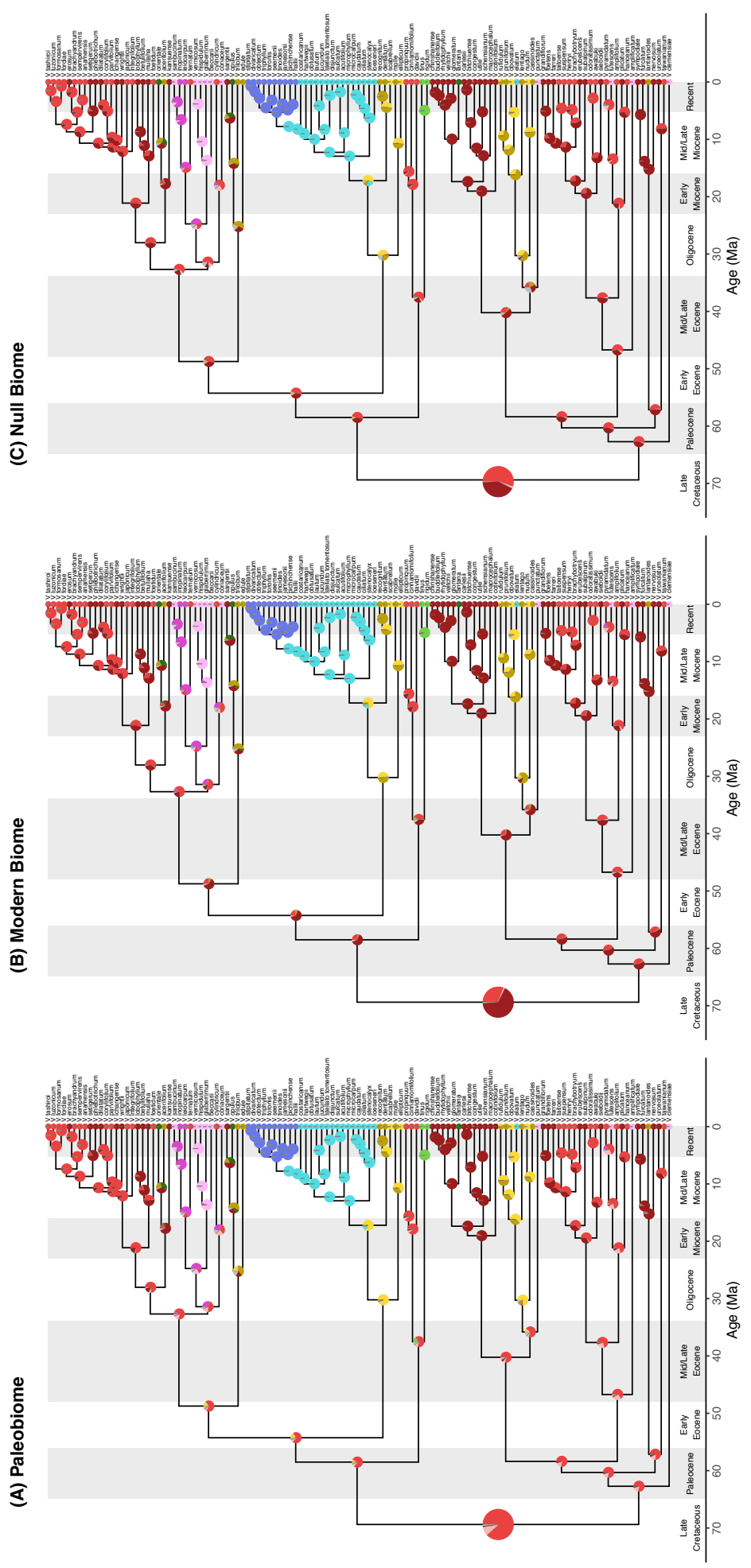

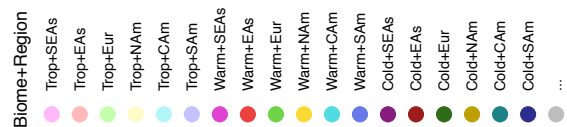


bioRxiv preprint doi: https://doi.org/10.1101/832527; this version posted June 1, 2020. The copyright holder for this preprint (which was not certified by peer review) is the author/funder, who has granted bioRxiv a license to display the preprint in perpetuity. It is made available under aCC-BY 4.0 International license.

\section{Figure 6}
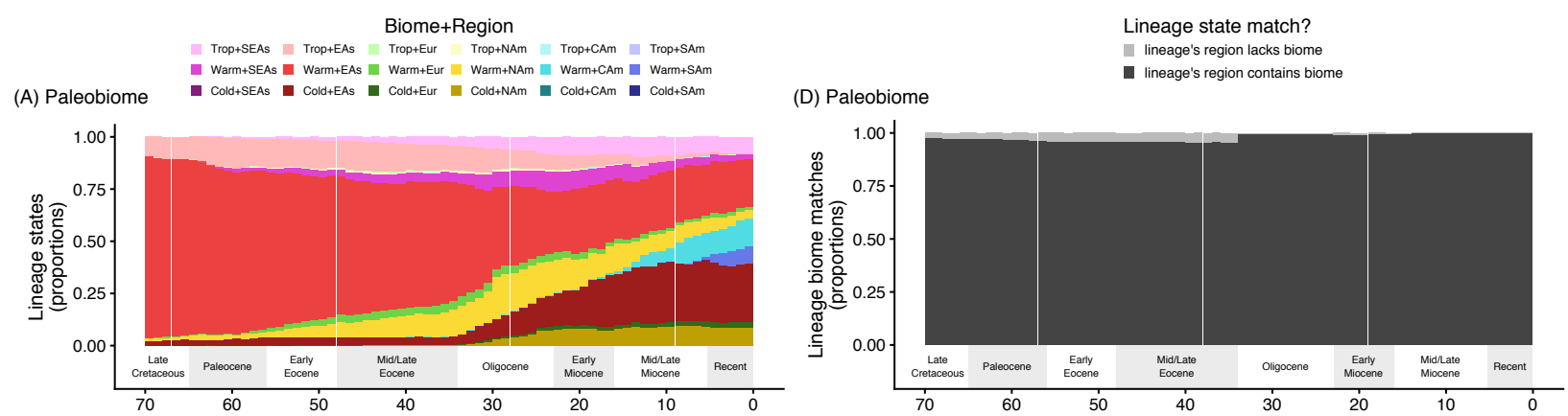

(B) Modern Biome

(E) Modern Biome
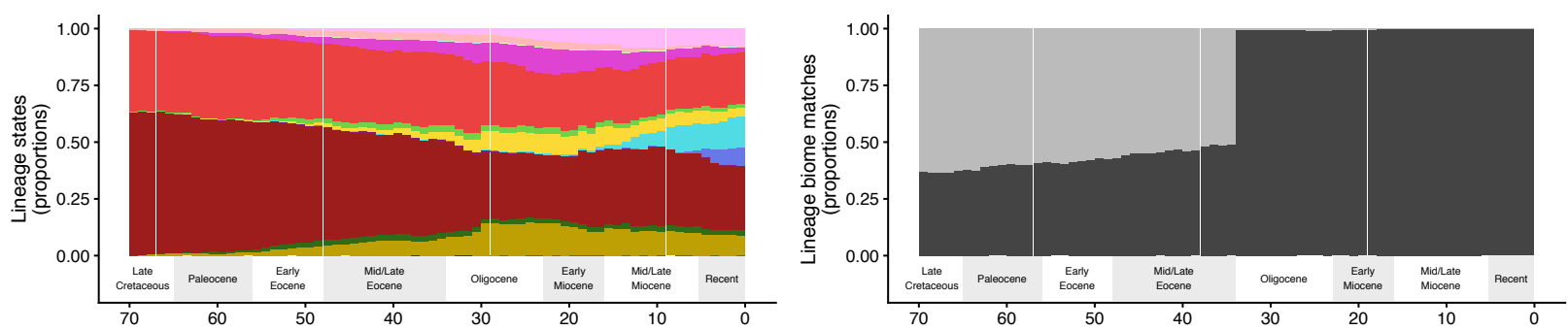

(C) Null Biome
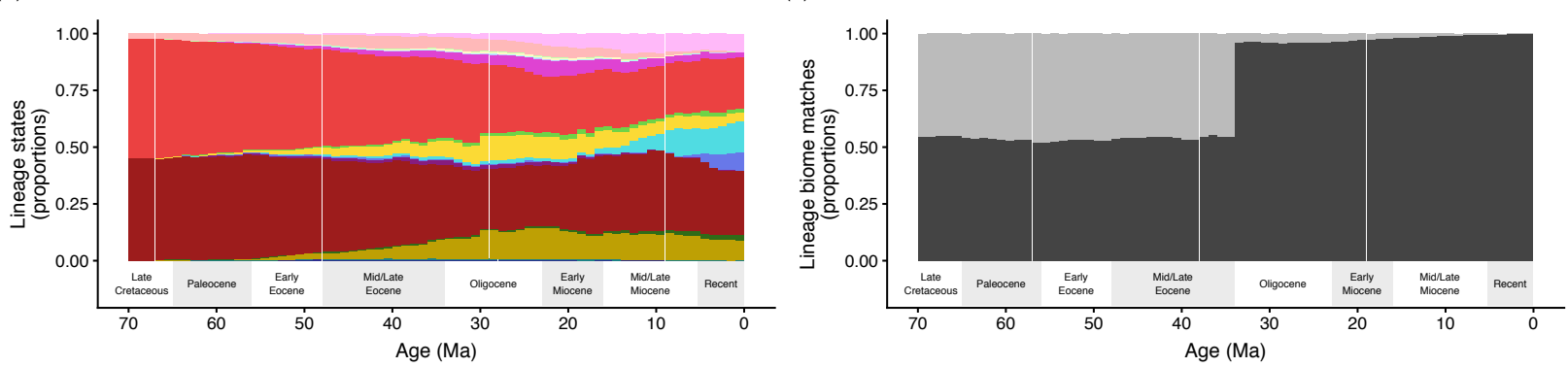
bioRxiv preprint doi: https://doi.org/10.1101/832527; this version posted June 1 2020. The copyright holder for this preprint (which was not certified by peer review) is the author/funder, who has granted bioRxiv a license to display the preprint in perpetuity. It is made available under aCC-BY 4.0 International license.

\section{$1344 \quad$ Figure 7}
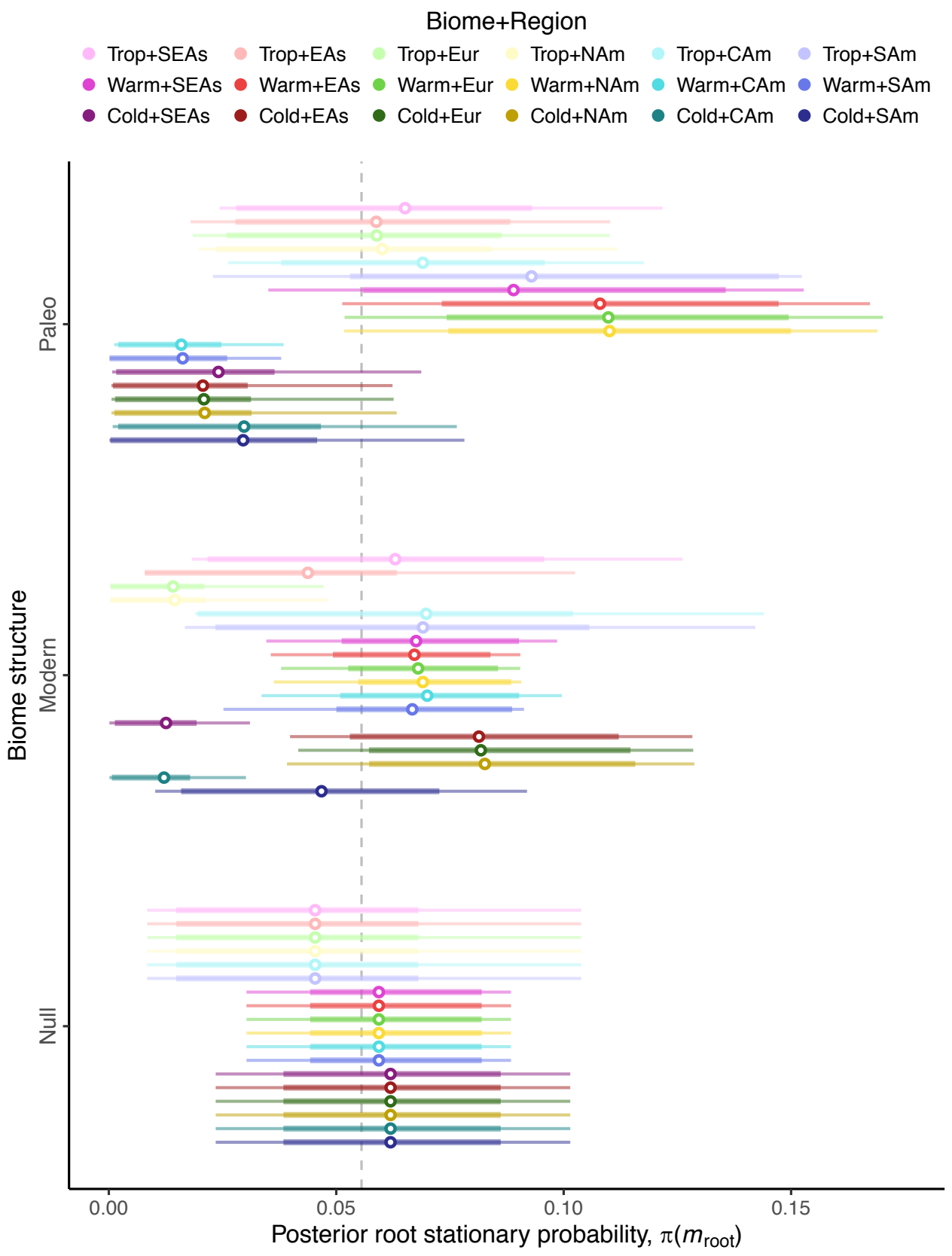

1345 
bioRxiv preprint doi: https://doi org/10.1101/832527; this version posted June 1,2020. The copyright holder for this preprint (which was not certified by peer review) is the author/funder, who has granted bioRxiv a license to display the preprint in perpetuity. It is made available under aCC-BY 4.0 International license.

\section{Figure 8}
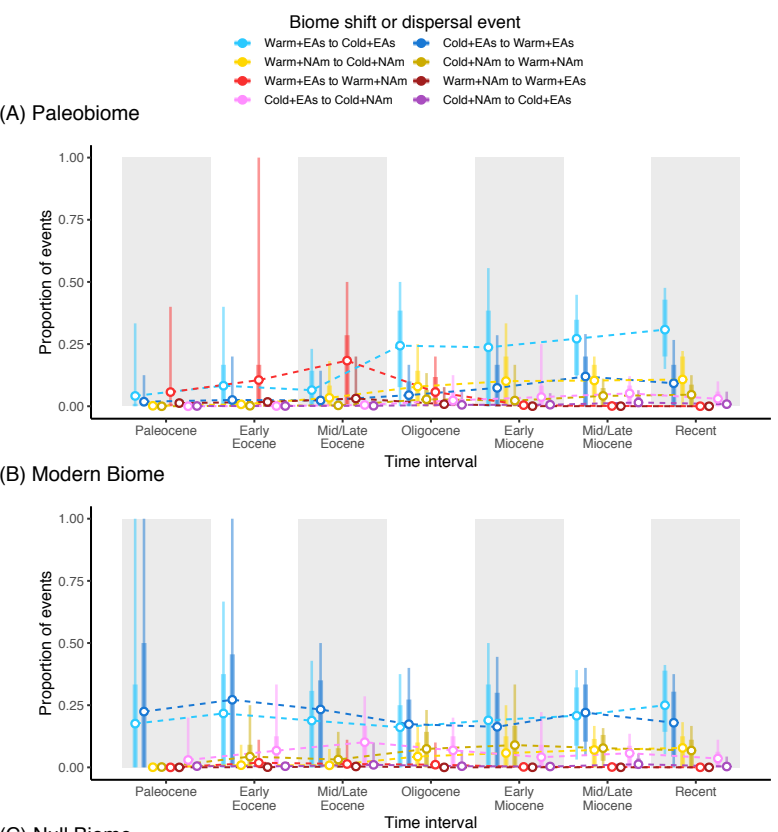

(C) Null Biome

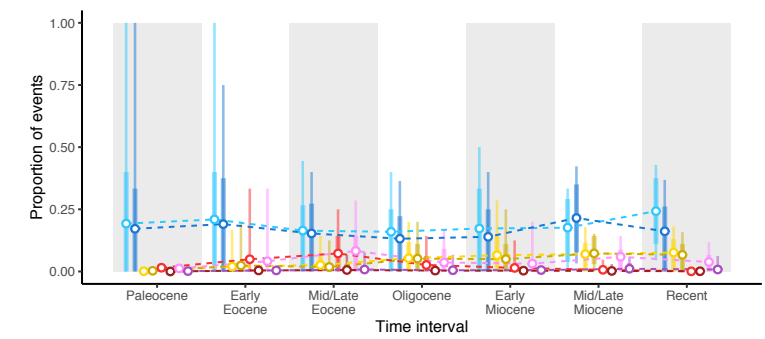

Event series type

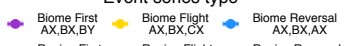

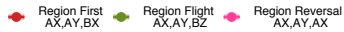

(D) Paleobiome

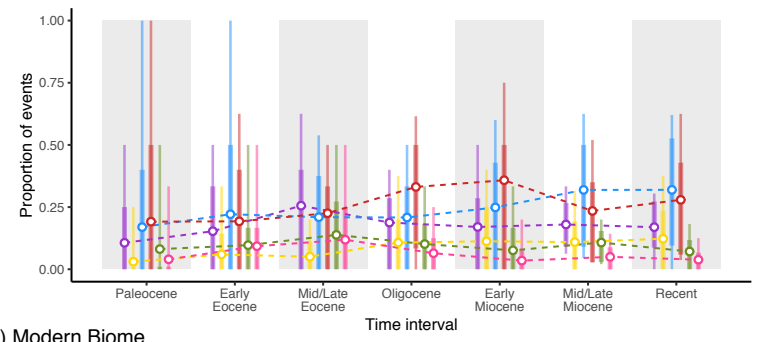

(E) Modern Biome
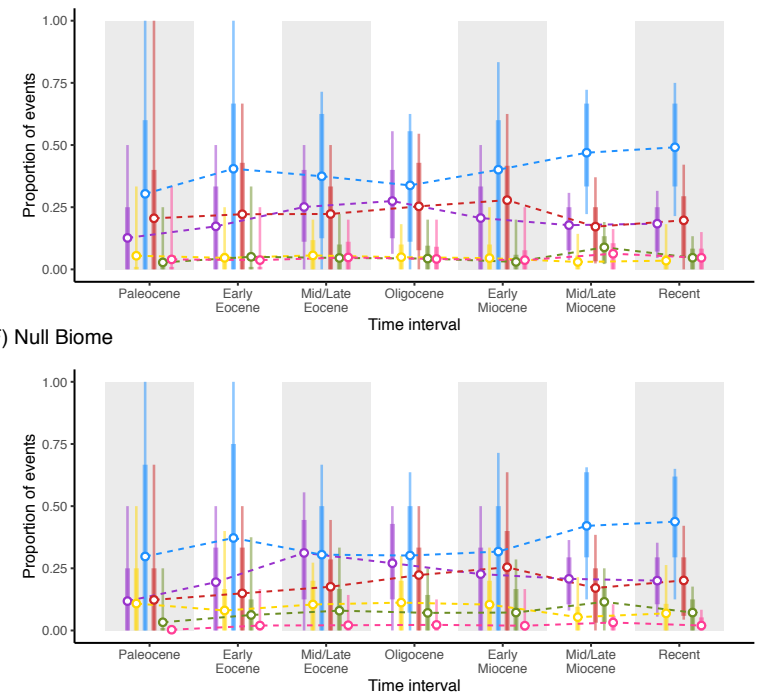